\title{
Capgemini Consulting
}

\section{DIGITAL TRANSFORMATION: A ROADMAP FOR BILLION-DOLLAR ORGANIZATIONS}

FINDINGS FROM PHASE 1 OF THE DIGITAL TRANSFORMATION STUDY CONDUCTED BY THE MIT CENTER FOR DIGITAL BUSINESS AND CAPGEMINI CONSULTING

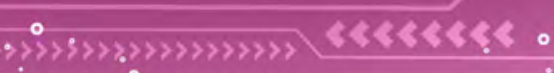
0 


\section{Contents}




\section{Common pressures; different results}

08

- Common pressures to begin digital transformation

- Different digital transformation speeds and different results

Case Study: Finding new opportunities for digital transformation

across a hospitality company

Case Study: Digital transformation in India

\section{The shape of digital transformation}

- Transforming customer experience

- Transforming operational processes

- Transforming business models

- Digital capabilities

Case Study: Digital transformation in an international banking group Case Study: Moving from separate to integrated digital initiatives in an apparel manufacturer

\section{Digital technology usage}

\section{Challenges on the road to transformation}

- Initiation challenges

- Execution challenges

- Governance challenges

Case Study: Evolving as a value chain orchestrator in mortgages

Case Study: Digital transformation in an international airport

\section{How can you make your digital journey successful?}

- Envision the digital future for your firm

- Invest in digital initiatives and skills

- Lead the change from the top

Case Study: Driving digital transformation across a global media company

\section{What is digital transformation maturity?}

58

- The What and the How

- Digital Maturity Matrix

- How to assess your digital maturity

Case Study: Capitalizing on digital opportunities for a business directory

\section{Conclusion}




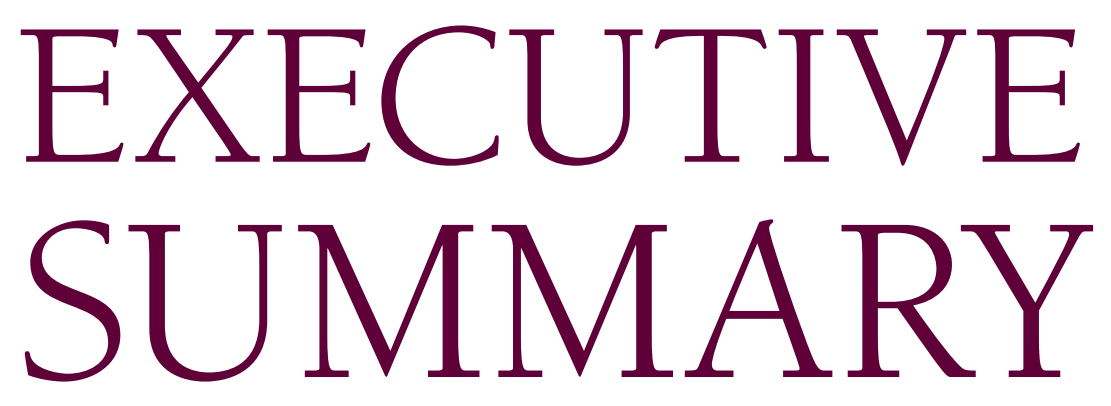




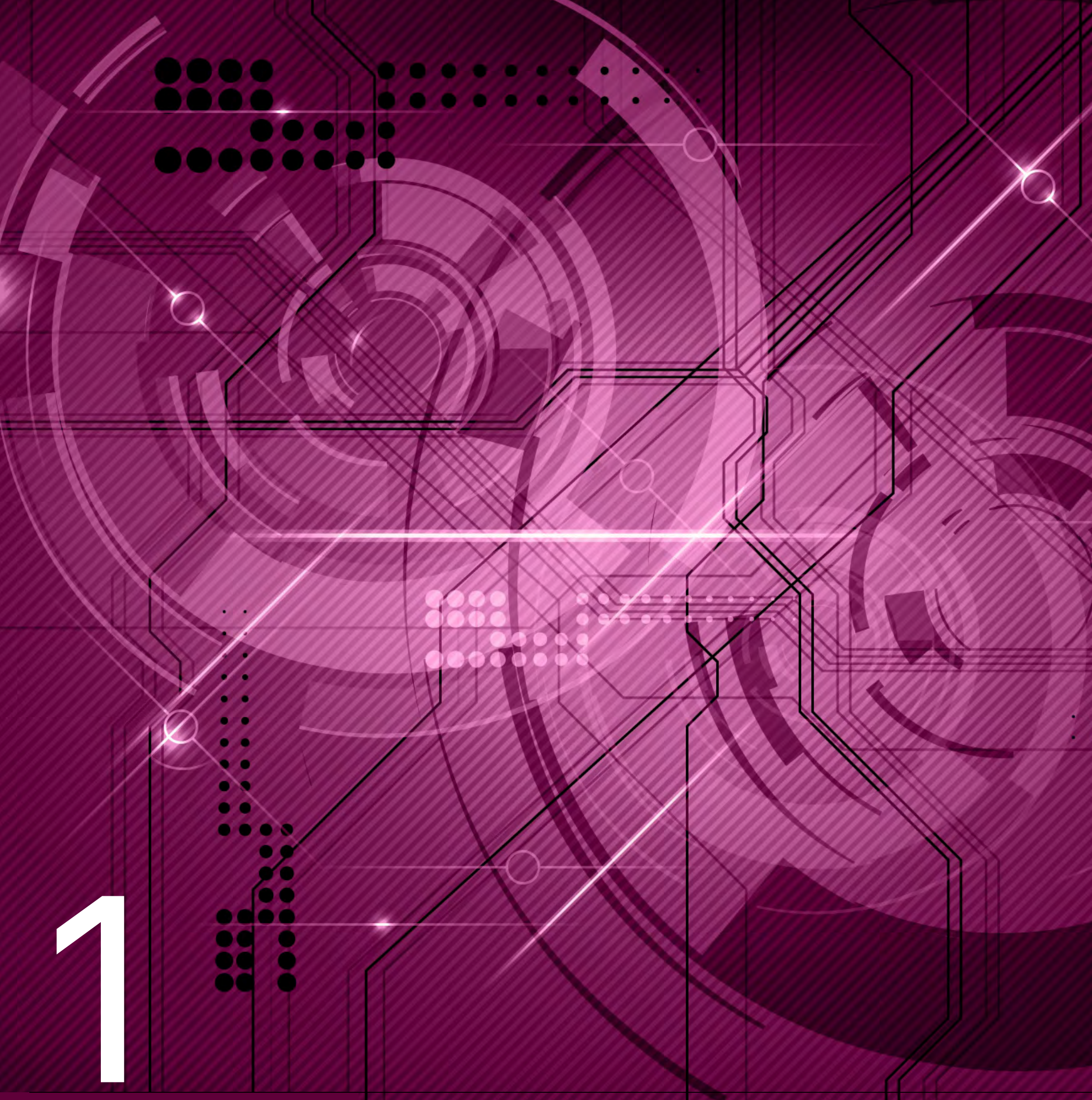

\section{THE STUDY}


Many popular stories of digital transformation involve digital startups or high-tech firms such as Amazon, Apple, or Google. Executives in traditional companies often find these examples hollow. Big traditional companies, with decades of history and legacy, are simply different from these newer digital entrants. Other stories focus on exciting initiatives by traditional companies, but are anecdotal and do not show how those companies implemented the change. These examples may be good triggers for other companies to take action, but they give no advice on how or whether to do so.

With that in mind, MIT Center for Digital Business and Capgemini Consulting set out investigate the ways in which large traditional companies around the world are managing
- and benefiting from - Digital

Transformation. We are working to understand how digital technology is changing the business of large leading firms.

Phase 1 of this multi-year study is an exploratory investigation involving 157 executive-level interviews in 50 companies in 15 countries (see Figure 1). These companies are large, with typically $\$ 1$ billion or more in annual sales. We conducted in-depth interviews to qualitatively explore the nature of the digital transformation phenomenon with real executives in real companies. To provide balanced perspectives, approximately half of the interviewees are business leaders such as CEOs, line of business managers, marketing heads, or COOs, while the other half are IT and technology leaders.

Figure 1: Interviews cover a broad range of countries and industries

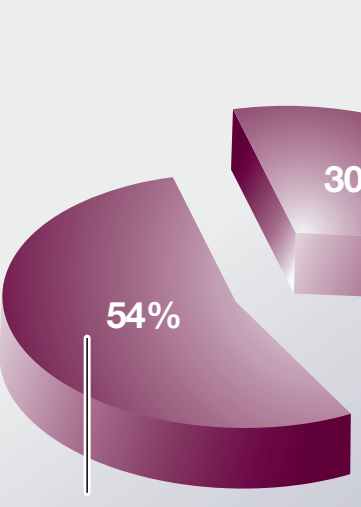

Europe

\section{Americas} \& Canada
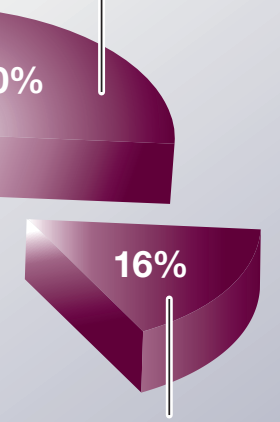

Asia Pacific

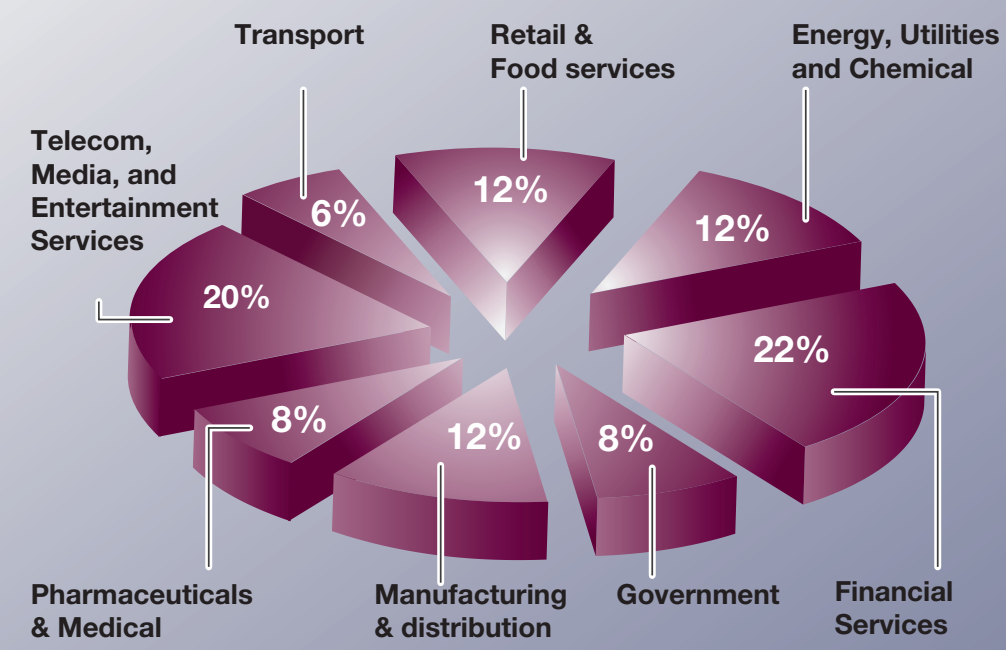




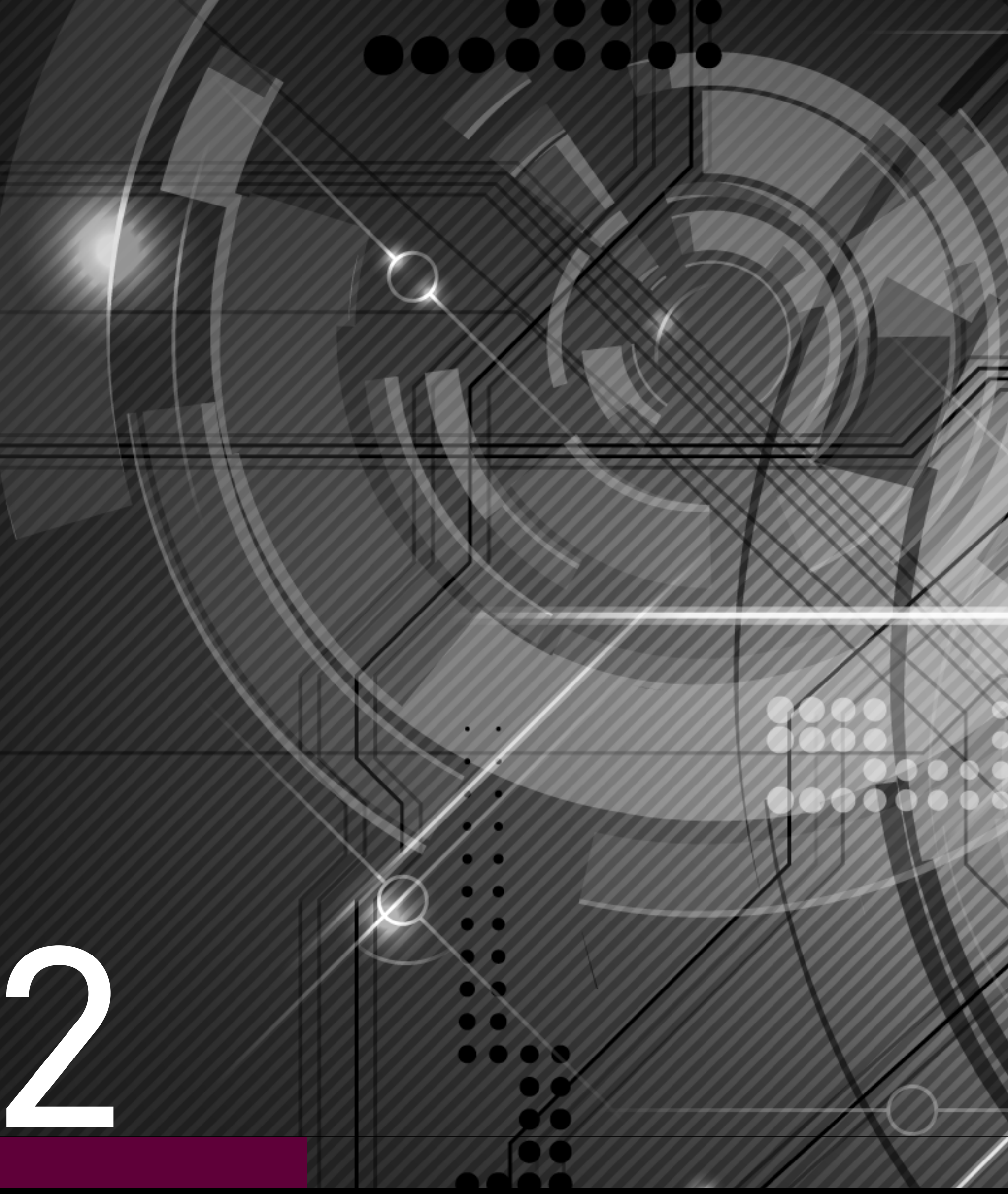

COMMON PRESSURES; DIFFERENT RESULTS 


\subsection{Common pressures to begin digital transformation}

The executives we interviewed report feeling pressure to transform from forces inside and outside the company. Figure 2 shows that most executives expressed at least moderate pressure to change. Employees are asking why they cannot collaborate with coworkers the way they can with friends. One executive quoted younger employees as saying "Come on. I know the company's more than 100 years old, but our IT capabilities don't have to match the age of the company." Other employees are incorporating their personal practices into their work lives without waiting for corporate practices to catch up. According to one CIO, "Officially iPads are prohibited by our policies. Yet 50 percent of our employees are using them at work."
Customers, too, are becoming more demanding. An airport executive described an "ever-rising tide of customer expectations" for service and convenience. For a manufacturer/ retailer, "People's expectations have changed. They want to be more in charge. They expect that we know them more... their online behavior is developing expectations and behaviors that they apply in other contexts as well."

Nearly every executive ( 72 percent) cited competitive pressures to change. This relates closely to increases in the pace of business. Respondents rated the current pace of business relative to five years ago as 5.6 on a scale from $1=$ very much slower to $7=$ very much faster. Most indicated the pace will continue to increase, leading to further pressure to transform their businesses. While several executives indicated this was "the new normal" for their

*Scale: $1=$ very much slower than 5 years ago, $7=$ very much faster than 5 years ago

\section{Figure 2: Pressures for change*}

High

Medium
$70 \%$

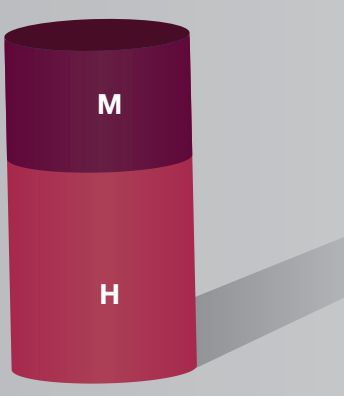

Customers

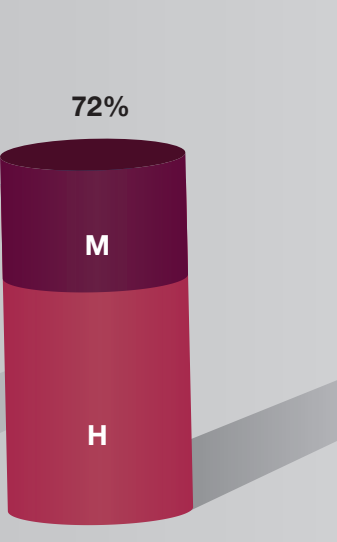

Competitors
$32 \%$

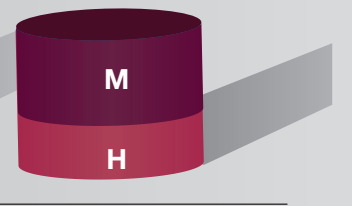

Employees

*Percentage of companies experiencing moderate or high pressure to transform from customers, competition, or employees as mentioned by their executives. Some firms mentioned more than one pressure. 
industries, others expressed concern that the acceleration would not be sustainable.

Executives on average rated their innovation culture at only 4.2 on a 7-point scale. Although few companies strive to be as high as seven on the scale, none of the executives felt their innovation culture was as strong as it should be.

Some executives expressed other, less common, pressures. Globalization is a driver for seven firms. These companies have a strategic need to expand globally or are facing pressures to generate information and process synergies across their global enterprises. Executives in three firms mentioned $\mathrm{M} \& \mathrm{~A}$ as a pressure to transform.

\section{Executives rated their innovation culture as 4.2 on a 7-point scale.}

\subsection{Different digital transformation speeds and different results}

A few companies in our study are using digital technologies, old and new, to truly transform their businesses. However, these companies are in the minority.

Despite internal and external pressures, very few businesses are gaining the full value of technologically-enabled change, even with technologies they already own. Many companies we interviewed took on the significant effort and expense of implementing ERP, CRM or other technology-enabled changes, but obtained only basic levels of value from the investments. They can obtain much more value by continuing the effort - envisioning new capabilities and process changes to reap larger returns from their foundational investments. Manufacturers' ERP systems have improved process efficiency and are providing better financial data for strategic decisions, but companies have not yet retrained buyers in how to use the information in negotiating with suppliers. Retailers and other service firms are gathering detailed information on buyer behavior, but lack the analytics capabilities to truly understand the power of the data. A hospitality company is conducting experiments in mobile marketing in two different business units, and building digitallyenhanced customer service apps in another, but is not conducting a unified digital transformation of the customer experience.

These companies are gaining some value from transforming parts of their businesses, but are only part of the way toward achieving the potential of digital transformation. Still others have not yet begun their digital transformation journeys.

In the following sections, we will describe how firms are digitally transforming, the challenges they are facing, and the levers executives can use to drive digital transformation. 


\section{Case Study}

\section{HOTELCO: FINDING NEW OPPORTUNITIES FOR DIGITAL TRANSFORMATION ACROSS THE ENTERPRISE}

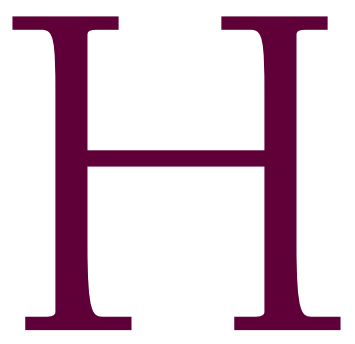

otelCo is one of the world's ten largest hospitality companies. The firm constantly strives to improve operational efficiencies and customer service both within and across properties. The firm's loyalty program is the centerpiece of the company's approach to understanding, serving, and selling to customers.

HotelCo executives, who have always believed in the power of technology to improve processes and increase customer intimacy, see further opportunity for digital transformation. Opportunities exist not only in new technologies such as social media and mobile, but also in the firm's more traditional areas like transactional IT and analytics. HotelCo is actively pursuing digital transformation in many areas and is working to innovate further. Because HotelCo experiences organizational and IT challenges common to many large global enterprises, it seeks to take a rational, well-defined approach to its digital transformation initiatives wherever possible.

HotelCo has created opportunities for digital transformation in three areas: online presence, mobile customer engagement, and internal operational processes.

Online: HotelCo continually evolves its online presence. It has integrated the website presence of all its properties on a common platform, enabling marketing across properties within a market. It also added new capabilities. For example, property managers using the firm's corporate digital marketing tools can control their property content, e.g., events, independently of other properties, while not having to build and maintain their own online capabilities. Further extending online capabilities, HotelCo has begun to implement social media and mobile marketing capabilities. Employees actively monitor social media for negative information and can respond. However, organizational questions remain about how to improve the response capability as volumes continue to grow: "Who handles it? Marketing? Product support? Customer support?"

In addition, HotelCo is integrating social media information onto its websites. The website allows user comments on select pages, and the firm is working to extend comments to additional pages. HotelCo is also incorporating third-party social media onto the site. For example, tripadvisor.com ratings feed directly into property web pages, despite the initial hesitation of some property managers. "First off, this conversation is happening and most of the customers are now savvy enough they're going to tripadvisor anyway. Let's bring the content right to our site, positive or negative. Customers see that and they say 'OK, well this 
is an honest assessment from people like myself...." The marketing people have found that such content is much more compelling than internally generated information.

Mobile: Realizing that most customers now carry cell phones, HotelCo has made mobile a key part of its marketing strategy. The company offers multiple smartphone apps and is making preparations for a future in which more people access sites via mobile than from a personal computer.

HotelCo actively engages in mobile marketing. The company realizes the customer intimacy it can achieve through mobile because customers react to messages almost instantly. SMS and WAP sites are a core part of mobile marketing because of their ubiquity. The different properties are in charge of their own mobile marketing messages, but the messages are delivered through a unified corporate-level system.

The company is also experimenting with location-based services. Innovation activities that initially focused on embedded devices and RFID have been facilitated by mobile phones. For example, customers arriving at some properties can receive mobile coupons for restaurants, shows, and other services in the hotel. Customers can also receive personalized service and information from digital displays located around the properties using phones or their loyalty cards.

While mobile and location-based services show promise, they have challenges. The firm is trying to balance a tradeoff between the capabilities of location-based devices and their potential intrusiveness. For example, when building a digital customer service display, HotelCo opted to ask customers to place their loyalty cards on the device rather than have the device automatically read who they are.

Additional challenges are technological and organizational. The firm wants to "support experimentation by all parties, but also clearly delineate their roles and scopes." For example, multiple groups conducting mobile experiments meet regularly to coordinate activities. And the company is starting to examine how it coordinates its use of vendors so that knowledge is not fragmented across multiple digital transformation initiatives.

Internal operations: HotelCo is investigating how to incorporate emerging technologies into internal processes. Compared with the device-agnostic policy for customers, HotelCo aims to use standard devices for internal processes and to make them available only to those employees who need mobile capability for their jobs.

The firm is trying to take the same measured process to implementing social media in internal processes as it does in other areas. One obstacle stems from security and cost: free platforms are not seen as secure, and the firm wants to be careful about investing large amounts of money to in license a secure platform for an uncertain goal. Another challenge is organizational: who would own the internal collaboration process? Until there is a clear internal owner and a 
well-defined purpose, the firm is taking a slow approach to widespread internal use of social media instead of targeted efforts.

The firm is midway through a set of initiatives aimed at rationalizing processes across the enterprise. When complete, HotelCo will have a set of shared services that are available to all properties, but which can be tailored by properties for their own use, much as the online platform is today. IT, while a significant enabler of the firm's capabilities, remains a challenge as the company changes. For example, the loyalty database is not optimized for multi-channel interactions, making it difficult to integrate information that arrives from, or is sent via, multiple media. Another challenge is integrating information and processes from acquired companies into the firm's core IT backbone.

At HotelCo, analytics is "something we do a ton of." Executives are proud of the firm's ability to understand drivers of customer and operational metrics. HotelCo is now investigating how to move analytics capability further out to the edges of the company. However, executives expressed the desire to make good choices about where to apply analytics. Analytics is "absolutely a different way of thinking... we don't need every role to do it, but we definitely want the skills in certain places. "For example, analytics may enable the firm's customer-facing employees to further personalize the customer experience, but executives are aiming to do it in a way that doesn't disempower those workers.

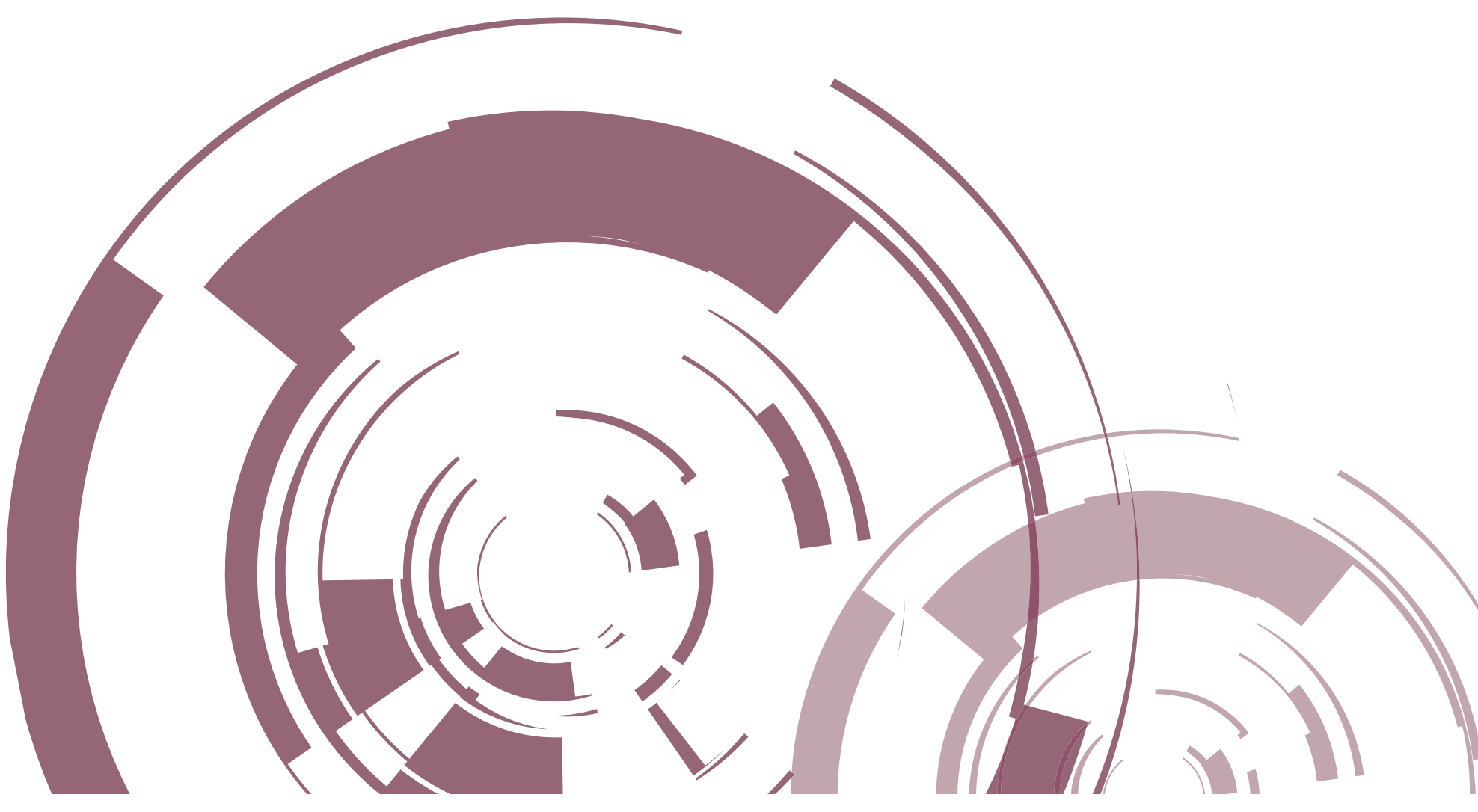


Case Study

\section{ASIAN PAINTS: DIGITAL TRANSFORMATION IN INDIA}

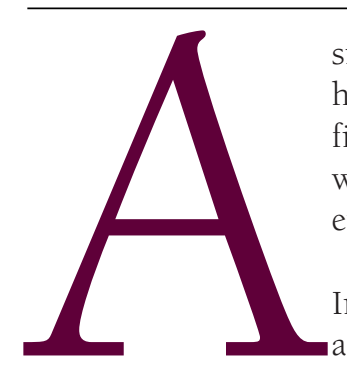

sian Paints, India's leading manufacturer of coatings, has digitally transformed its business step by step. The firm has been able to maintain fast growth and globalize while increasing efficiency and reducing its impact on the environment.

In the early 2000s, as part of an effort to reduce debt and increase internal efficiencies, the firm implemented an enterprise-wide ERP and advanced Supply Chain

Management system. This helped to create an enterprise-wide platform that was the basis for further improvements in sales and customer processes. The change also liberated working capital that helped fund acquisitions in emerging markets. Asian Paints gained further efficiencies by linking subcontractors and suppliers on a B2B portal. In 2003, the firm invested in a CRM system.

In 2010, Asian Paints centralized its order taking process into a single corporate call center. This change helped the company further improve operational efficiencies and sustain its growth. In customer-facing processes, the move entailed much more than just creating the call center. Retailers were encouraged to place orders through the call center, where they could receive a more consistent service level than they could through the firm's 100 local depots. Centralized data also enabled delivery of products to large institutional customer job sites, giving the company a capability that competitors could not provide.

The biggest change resulting from centralizing order taking was in the way salespeople interacted with retailers. Liberated from routine order collection, the sales team could focus on building stronger relationships. To enable a more meaningful dialogue between the sales team and each retailer, the company provided salespeople with vital customer data in the field using mobile devices. A rollout of tablet devices, which is currently underway, will further mobile-enable the sales staff.

Asian Paints' digital transformation also extended to internal production processes. High growth in paint demand creates the need to set up new manufacturing plants every three years. Given the scale and size of these plants, the firm has begun to focus on creating highly automated plants. Automation has led to greater scalability, better quality and stronger safety and environmental protections. The new plants are fully integrated from an information management perspective. Data from shop floor control systems and automated warehouses are linked seamlessly to the ERP. This has helped to further sustain the firm's operational efficiencies. 
In India, Asian Paints has begun to sell services (such as a painted wall) instead of products (a can of paint). The services strategy has benefits beyond new revenues. Selling services helps to ensure that high-end products are applied properly, thereby improving customer satisfaction. Services also help the firm to get closer to the end-consumer, understand core consumer choices, and launch highend texture finishes. Additionally, having a contractor in a client's house yields information that marketing staff can use to understand both customer preferences and potential demand. Gaining better knowledge of end-customer preferences is a high priority for the company, since its business model - operating largely through retailers - means that customer involvement has been traditionally very low.

The relationship between IT and business executives has traditionally been very strong. The IT team consists of business graduates with a deep understanding of the underlying business processes. They act as internal process consultants. Governance is centralized and top down, with a strong executive-level understanding about the importance of standardization and coordination. Although business executives suggest many digital investments, IT executives originate others such as the firm's new analytics initiative.

Corporate growth and new technology demands have increased the challenges of digital governance. The firm is in the first year of a new portfolio planning process. "We have enjoyed great equity with the business because we have delivered solutions. But seeing so many projects coming at us, we felt that now we need to establish processes to match expectations and resources whilst taking on innovative projects," says Mr. Manish Choksi, $\mathrm{CIO}$ and Chief of Strategy.

Asian Paints' investment in strong IT capabilities, combined with organizational and process changes intended to leverage those investments, will serve as a foundation upon which the organization can continuously envision and implement new forms of digital transformation. 


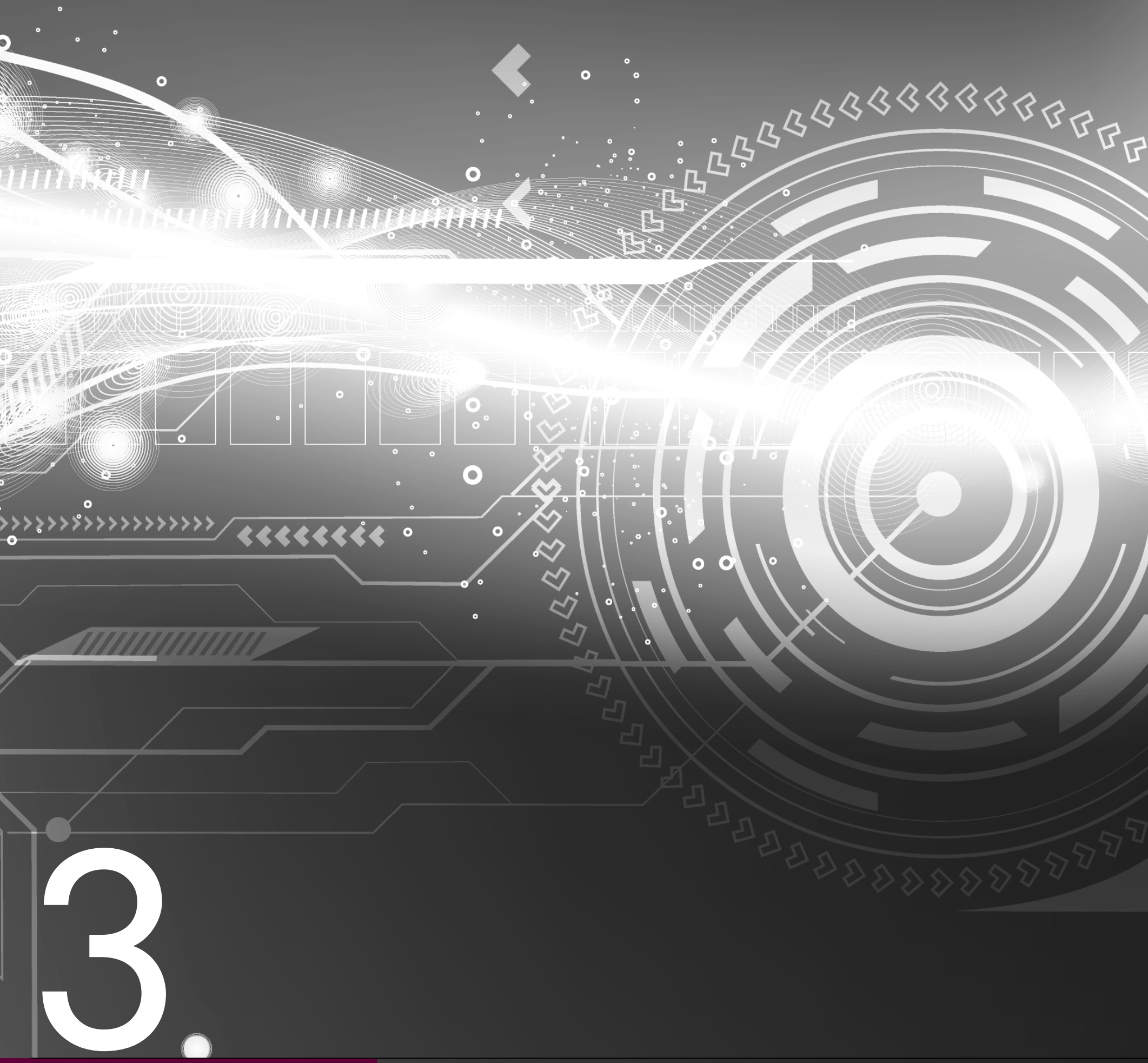

THE SHAPE OF DIGITAL TRANSFORMATION 
The companies interviewed are moving forward with digital transformation at varying paces and experiencing varying levels of success. Some are transforming many parts of their organizations while others are still doing only the basics. Others are encountering organizational issues or other challenges that prevent them from transforming successfully.

Analysis of the interviews shows clear patterns. Executives are digitally transforming three key areas of their enterprises: customer experience, operational processes and business models (see Figure 3). Within each of the three pillars, different elements are changing. These nine elements form a set of building blocks for digital transformation. Currently, no company in our sample has fully transformed all nine elements. Rather, executives are selecting among these building blocks to move forward in the manner that they believe is right for their organizations. The tenth elementdigital capabilities - is an essential enabler for transformations in all areas.

In this section, we explore how companies in our study are implementing each transformational building block. Subsequent sections examine adoption of four key technologies and then common

\section{Figure 3: Building blocks of the digital transformation}

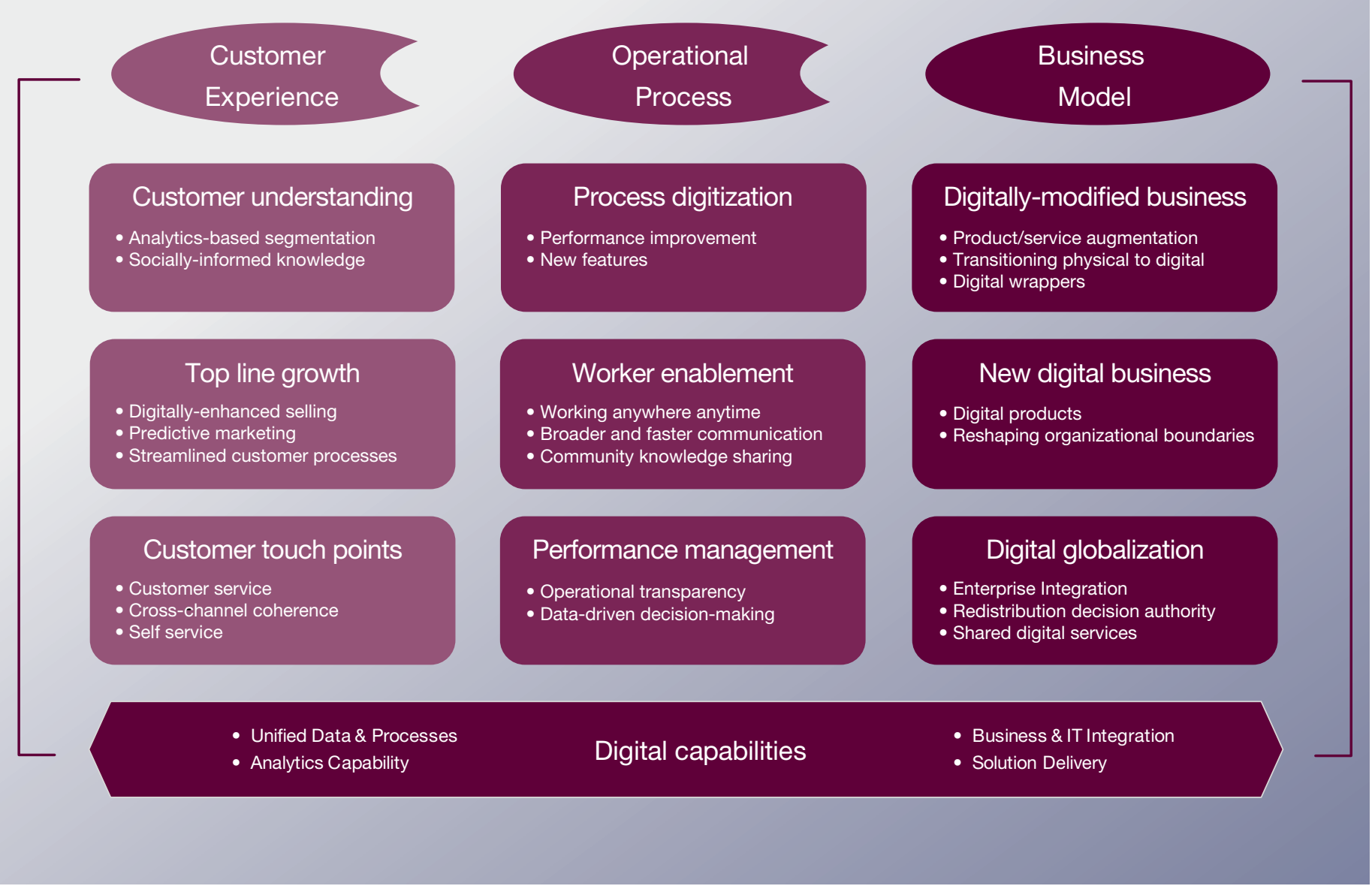


challenges that interfere with digital transformation. The paper concludes with a set of key levers senior executives can use to successfully transform their organizations.

\subsection{Transforming customer experience}

\section{Customer understanding}

Companies are starting to take advantage of previous investment in systems to gain an in-depth understanding of specific geographies and market segments.

Other firms are exploring social media to understand customer satisfiers and dis-satisfiers. A mortgage executive noted "We launched a non-profit real estate community to help our customers. As an unplanned benefit this helps us to understand them better. We launched special products, such as a special loan offering for divorced people, following several customer questions."

In addition, companies are learning to promote their brands more effectively through digital media:

- A medical device firm is launching a social media campaign to promote a radically new class of products in an online community of medical specialists. Executives expect awareness to grow in weeks rather than years.

- A media company has teamed with product manufacturers for social media-based brand enhancement tied to in-person events.

- After the 2009 economic downturn, a financial firm built a set of educational digital tools aimed at helping clients invest more safely. These tools, while not selling the firm's products directly, aligned closely with the firm's reputation for conservatism and safety.

Companies are also building new online communities to advise and build loyalty with clients in medical, real estate or financial services products. Others are building products that improve branding in lifestyle communities. A consumer sports equipment manufacturer enables individuals to publish their sport performance to communities and stay connected with other customers who have similar interests.

Many firms are building analytics capability to understand customers in more detail. Some insurance firms, for example, are improving their portfolios and cost structures through analyticsbased underwriting and pricing. Other companies are conducting analyticsbased experiments to drive customer behavior. In one case, a restaurant firm is actively conducting experiments in pricing and promotion across a set of franchised stores. The experiment dynamically adjusts product prices in response to demand, weather, inventory levels, and proximity to closing time.

\section{Top-line growth}

Companies are using technology to enhance in-person sales conversations. Financial services firms are using tablet-based presentations instead of paper-based slide decks to make sales pitches. Some are going beyond. Insurance firms are introducing mobile tools to help sales people and customers engage in analytics-based planning. 
However, problems remain as, for example, one retail executive described customers being angry that customer service representatives in a store could not access online order history.

Some firms are moving beyond simple multi-channel models to enable new forms of digital strategy. A mortgage company, for example, is offering $\mathrm{B} 2 \mathrm{C}$ offerings in one channel to complement its existing B2B offerings in another. It supplements the multichannel experience through a new type of storefront.

Several companies are offering selfservice via digital tools. These tools allow the customer to save time, while saving the company money. Classic mobile apps allow bank customers to access account information or media customers to have electronic rather than paper subscriptions. Many firms are now offering customer apps to enhance customer touch points. In a hospitality firm, smart phone apps are linked to the customer's profile, enabling integration across SMS, apps, and social media efforts. A media company offers apps with geolocalization and augmented reality to help customers find interesting places to visit and provide special offers via vouchers and e-couponing.

\subsection{Transforming operational processes}

Although transformed customer experiences are the most visible - and arguably the most exciting - aspects of transformation, firms are also realizing very strong benefits from transforming internal processes.

\section{Process digitization}

Companies historically have used automation to make processes more efficient and scalable. ERP for example has enabled significant efficiency and quality gains in core transactional, financial, and supply chain processes. An insurance firm has created a central digital platform for core claim processes and deployed the model across several countries. Employee self-service systems, especially in areas like HR, are also becoming widespread.

Some firms are going beyond simple automation to gain additional benefits. Automation can enable companies to refocus their people on more strategic tasks. A manufacturer has begun to centralize the HR function, allowing economies of scale through self-service while freeing HR people to "focus on enlarging manager skills, rather than counting days off." A specialty materials company has automated many R\&D processes. Automation allows researchers to focus on innovation and creativity rather than repetitive efforts. It also creates streams of data that can be useful in later data mining efforts.

New technologies are extending this trend of gaining benefits beyond efficiency. A paint manufacturer has created fullyautomated plants that significantly reduce labor requirements, improve product quality and enhance environmental, health, and safety performance. An apparel company has moved to digital design processes when collaborating with manufacturing partners. Going digital eliminates most need to ship physical prototypes back and forth, reducing the product development lifecycle by 30 percent. It allows the firm to be much more agile, changing designs quickly in response to market changes. 


\section{Worker enablement}

Once-novel technologies such as mobile e-mail, collaboration tools, and video conferencing have now become the norm in many companies. Employees routinely collaborate with people whom they have never met in person, in regions they have never visited. Mobile devices allow employees to stay connected with the office at all hours and to work from home when not able to be in the office. Individual level work has, in essence, been virtualized - separating the work process from the location of the work.

\section{Some companies are starting to} examine new possibilities for virtualizing individual work processes. A financial services firm rearranged its headquarters so that nobody had an assigned desk, even the CEO. Employees now work from home one or two days per week and, when they are in the office, sit near people with whom they are temporarily collaborating. Meanwhile, the firm's collaboration and networking tools allow employees to talk with anyone in the company from wherever they are sitting. This is setting the stage for further changes related to globalization.

In a broader sense, digital transformation replaces limited oneway vertical communication with broad communication channels that are both vertical and horizontal. $\mathrm{CxOs}$ can engage in 2-way communication quickly at scale. Employees can collaborate in ways that were previously not possible. The tools that virtualize individual work, while implemented for cost reasons, have become powerful enablers for knowledge sharing. Salespeople and front line employees, for example, are beginning to benefit from collaborative

\section{Figure 4: Digital transformation creates a virtuous cycle of knowledge sharing}

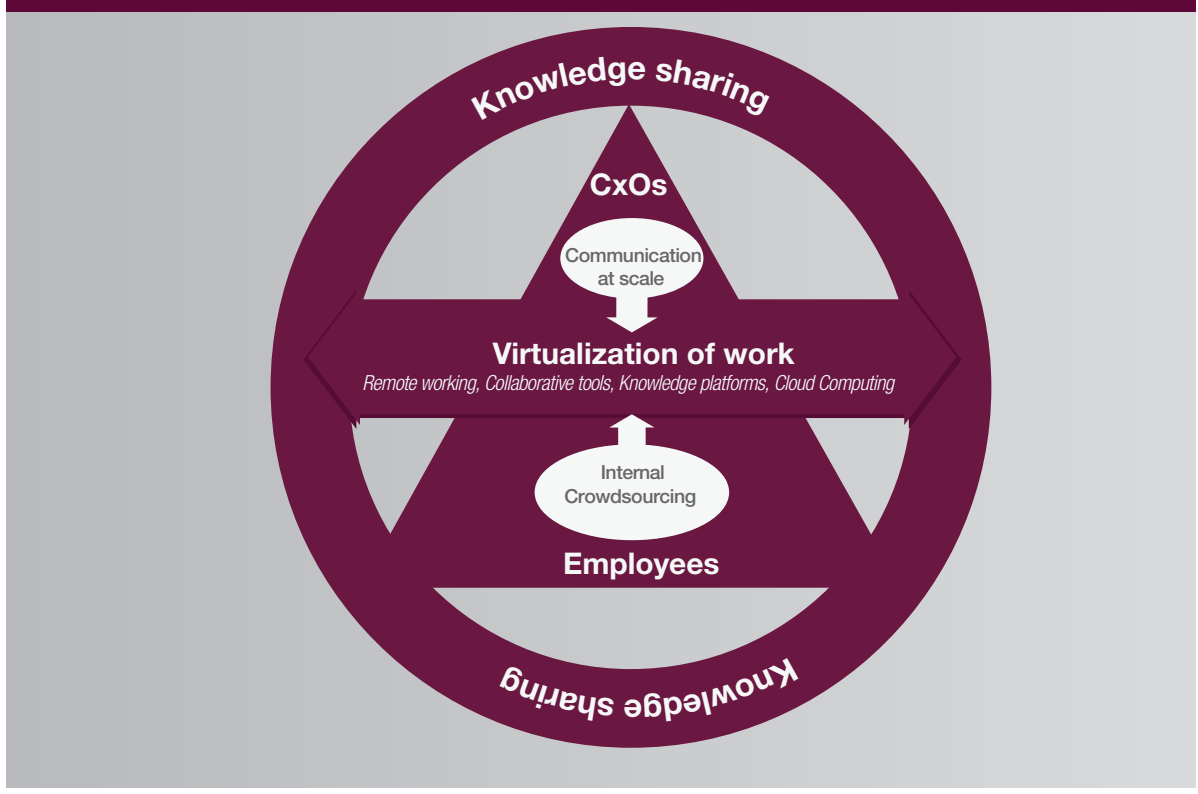


tools in which they can identify experts and get questions answered in real time. They are also increasingly gaining access to a single global view of the company's interactions with a customer.

\section{Performance management}

Performance transparency was a key highlight mentioned by several executives. Executives in most companies say they are more informed when making decisions. Transactional systems give executives deeper insights into products, regions, and customers, allowing decisions to be made on real data and not on assumptions. "Instead of simply revising last year's plan, we can use data to make better decisions about how we prioritize." This is happening in both internal processes and customerfacing processes. The level of detail is also increasing, allowing managers to compare status across sites or reallocate product manufacturing capacity in ways they could not do before. It is also prompting additional data gathering and integration as managers start to see the value of being better informed.

Beyond being better informed, digital transformation is actually changing the process of strategic decision-making. Top executives in a medical device manufacturer used the company's existing collaboration tools to extend strategic planning sessions from 12 people to more than 300 of the firm's top managers. This enabled better input into the process and better uptake of the vision after decisions were made.

\subsection{Transforming business models}

\section{Digitally-modified businesses}

A media executive said: "We've realized that if we don't transform the way we do business, we're going to die. It's not about changing the way we do technology but changing the way we do business." It is finding ways to augment physical with digital offerings and to use digital to share content across organizational silos. A grocery firm is staying true to its traditional business, but using digital to transform a new growth business. "After two years, our e-commerce platform is bringing us 20 percent of our new clients and our traditional clients are consuming 13 percent more on average."

Other firms are building digital or service wrappers around traditional products. A national post office is creating a free digital mailbox attached to each physical mail address that companies can use as a substitute for a person's physical mailbox. A business credit firm is developing a digital business for some credit products that requires less involvement than their traditional high-touch offerings.

\section{New digital businesses}

Companies are introducing digital products that complement traditional products. A sports apparel manufacturer started selling GPS and other digital devices that can track and report on a customer's workout. Other companies are changing business models by reshaping their boundaries through digital. A mortgage company is moving from being a link in the value chain to 


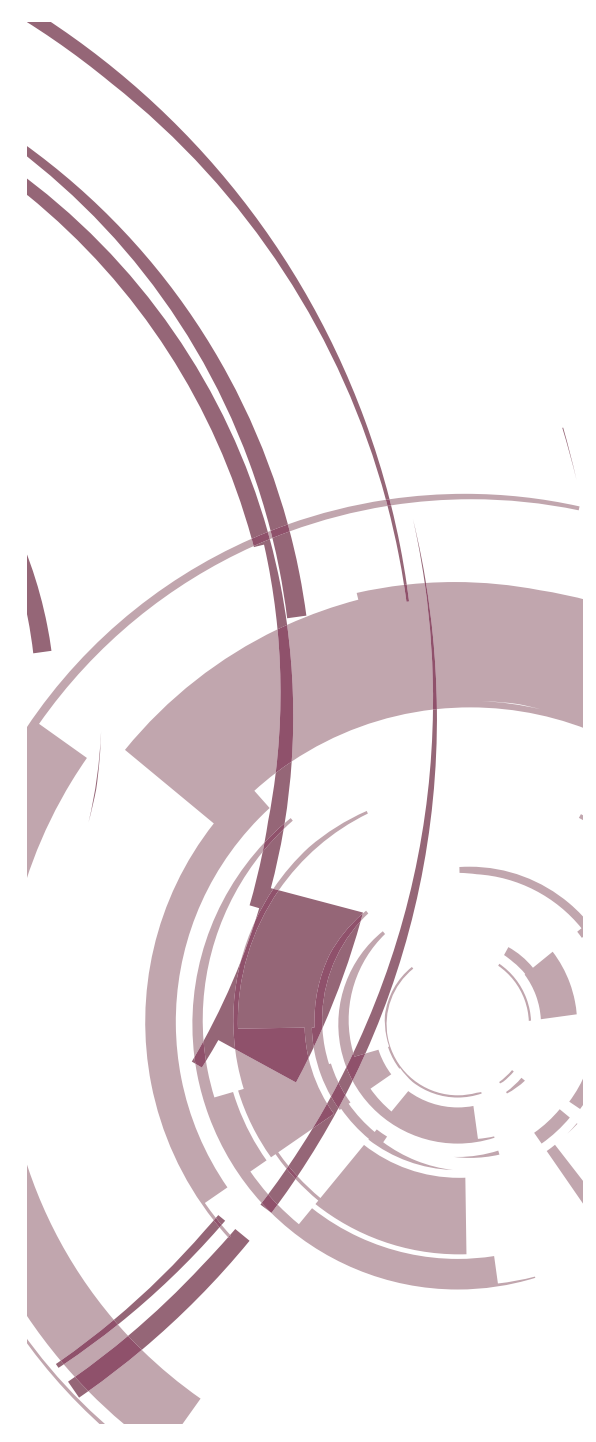

being a global assembler of investment products. An airport authority is aiming to become the owner of a traveler's end to end process. It will enable for travelers an integrated multichannel experience including information on airplane traffic and reservations, duty-free shopping promotions and other benefits.

\section{Digital globalization}

Firms are increasingly transforming from multi-national to truly global operations. Digital technology coupled with integrated information is allowing firms to gain global synergies while remaining locally responsive. They are, in the words of many executives, "becoming more centralized and decentralized at the same time." These companies benefit from global shared services for finance, $\mathrm{HR}$, and even core capabilities like manufacturing and design. Global shared services promote efficiency and reduce risk. They even promote global flexibility. One manufacturer can shift production around the globe with only a few days' notice in response to interruptions or excess demand.

For local managers, the pain of centralization is balanced by efficiency benefits and ability to focus on more strategic activities. Local managers, empowered to make decisions autonomously, also gain a broader view of the business through centralized data. They have the freedom to tailor business to their local needs, but the responsibility to act in the interests of the larger enterprise. Globalization also entails a different approach to policy: "fewer mandates from headquarters, but more guidelines."

\subsection{Digital capabilities}

Digital capabilities cut across all three pillars. They are a fundamental building block for transformation in customer experience, operational processes, and business models. Although CIOs and existing IT departments are leading digital initiatives across companies, they hire extra skills or implement separate units to coordinate digital transformation.

\section{Unified Data and Processes}

The most fundamental technology need for digital transformation is a digital platform of integrated data and processes. Large successful companies often operate in silos, each with their own systems, data definitions, and business processes. Generating a common view of customers or products can be very difficult. Without the common view, advanced approaches to customer engagement or process optimization cannot occur.

The difficulty of operating without a platform becomes greater as companies engage in multi-channel operations. Many companies, for example, cannot link customers' activity in stores or bank branches to their activity on the web or mobile. An executive in one company, echoing statements by many others, said "data integration is the biggest challenge in setting up our digital services: self-services, web-strategy and running partnerships."

Unified data and process is one reason that web-based companies are able to gain advantage through analytics and personalization much more readily than traditional firms. For many traditional companies, the first step in preparing for digital transformation 


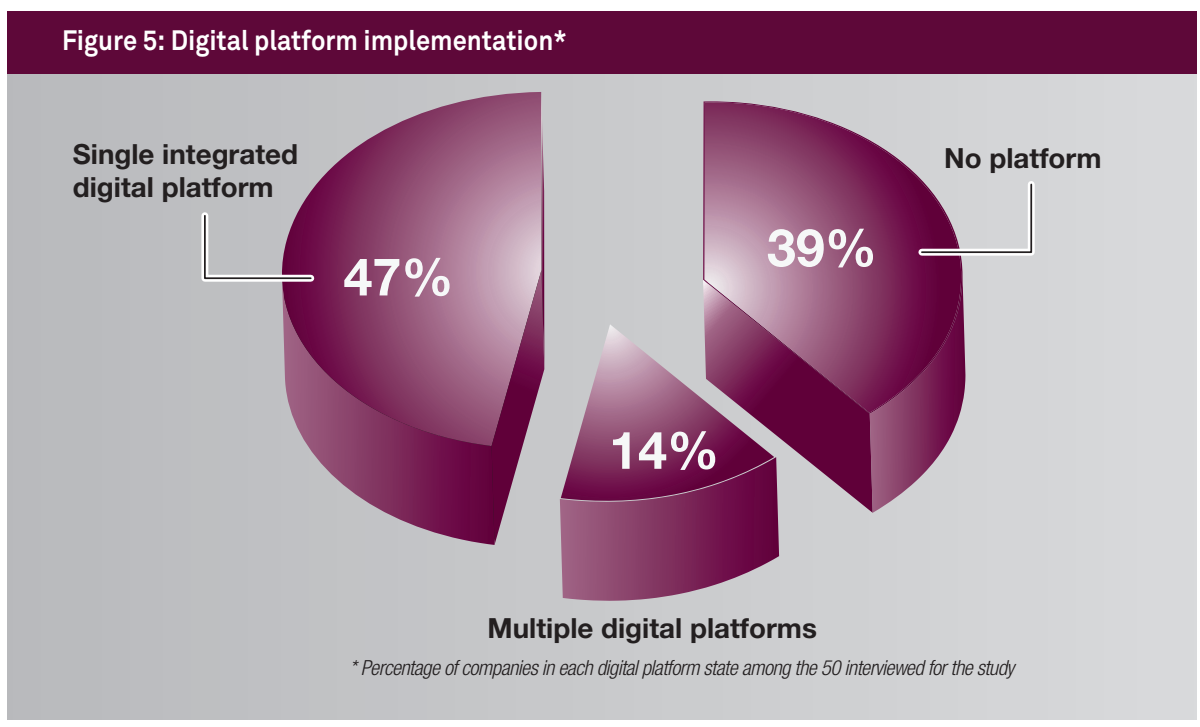

to invest - sometimes heavily - in integrating data and processes across the enterprise. In this respect, firms that have already implemented ERP and CRM systems are a step ahead of others.

Unified platforms can also help to manage the centralization/ decentralization challenge in globalization. Hospitality firm properties can use centralized platforms to deliver locally-customized information while not having to support the technology. A media company common technology platform allows the firm to share content at worldwide level, offering media content developed in one part of the world to be used easily in other markets and publishing formats.

\section{Solution delivery}

Companies also need the capabilities to modify their processes or build new methods onto the data and process platform. Solution delivery requires effective methods and strong skills. Most IT departments have solid development methods in place. However, those methods are often geared to well-defined requirements and mature technologies but not to emerging digital technologies and practices. Mobile and social media, for example, often require iterative approaches to learn about what will work in the market or workplace. They also use technology that is not commonly available in enterprises. Analytics activities often require specific knowledge and temperaments that typical IT developers do not possess.

Some IT departments have established special units to build emerging technology skills and methods. Others have innovation units to identify how new technologies and practices might change the business. Still others look outside the firm for help. 78 percent of the companies interviewed hire vendors to address large projects or those that require advanced technologies. Vendor partners can be very effective, but some executives expressed concern about over-reliance on vendors. A hospitality executive firm said that knowledge of key emerging technologies is spread across silos of external vendors, making integration difficult. Several executives described knowledge gaps that existed after they ended a vendor relationship. 


\section{Analytics capabilities}

Information management and analytics were highlighted as major goals by executives across industries. Several firms echoed the statement of an executive who stated "It's time to harvest the data and turn it into insights." Combining integrated data with powerful analysis tools is seen as a way to gain strategic advantage over competitors.

Companies are engaging in analytics at varying levels of intensity. Some are just beginning to make better use of the data available from transactional systems. They are making more-informed and better decisions and reacting more quickly to changes in their operations. Companies with integrated data are able to engage in more powerful analytics strategies. An Insurance company is able to do predictive sales, helping salespeople to target products that a specific type of customer is likely to buy. A hospitality company and a restaurant firm are using analytics to target promotions and conduct pricing experiments. A media company is using web analytics to provide real-time KPIs to customers in order to demonstrate the added-value of the firm's products. Several insurance firms are using analytics to underwrite policies, enabling better pricing and reshaping the firms' risk portfolios. However, all executives also mentioned that building analytics capability was difficult, requiring skills and culture change in addition to investments in information technology.

\section{Business and IT integration}

Digital Transformation, more than other business changes, requires strong integration between technology and business executives. Companies with a history of strained IT and business relationships are handicapped when trying to conduct digital transformation. These companies often have issues common to those that do not govern IT well, namely complex IT architectures, unintegrated data, and processes that are not well-enabled through technology.

In contrast, companies with a solid IT/ business relationship are in a solid position to begin digital transformation. A bank executive stated: "IT has been brought closer to business during the last five years. It is very important to success because many of the important transformations in our business enabled by technology." With trust and shared understanding, IT executives can help business executives meet their goals, and business executives listen when IT people suggest innovations. Where strong relationships exist, executives on both sides of the relationship are willing to be flexible in creating new governance mechanisms or digital units without feeling threatened.

In most firms, digital transformation is being led by CIOs. Where the relationship is strong, this approach can be very effective. At a manufacturer, the $\mathrm{CIO}$ is also head of strategy. He led aggressive investments in technology that generated tremendous benefits for the firm (see case study). The $\mathrm{CIO}$ of a global services firm also manages the firm's e-commerce operations and its innovation activities. Other companies place responsibility for digital in another unit or executive who works closely with the CIO. An apparel firm established a new digital division that works closely with the existing IT department. A business directory company hired a second $\mathrm{CIO}$ to focus on transformationrelated activities, working in concert with the $\mathrm{CIO}$ who focused on keeping the firms' existing platform functional. 


\section{Case Study}

\section{BNP PARIBAS: DIGITAL TRANSFORMATION IN AN INTERNATIONAL BANKING GROUP}

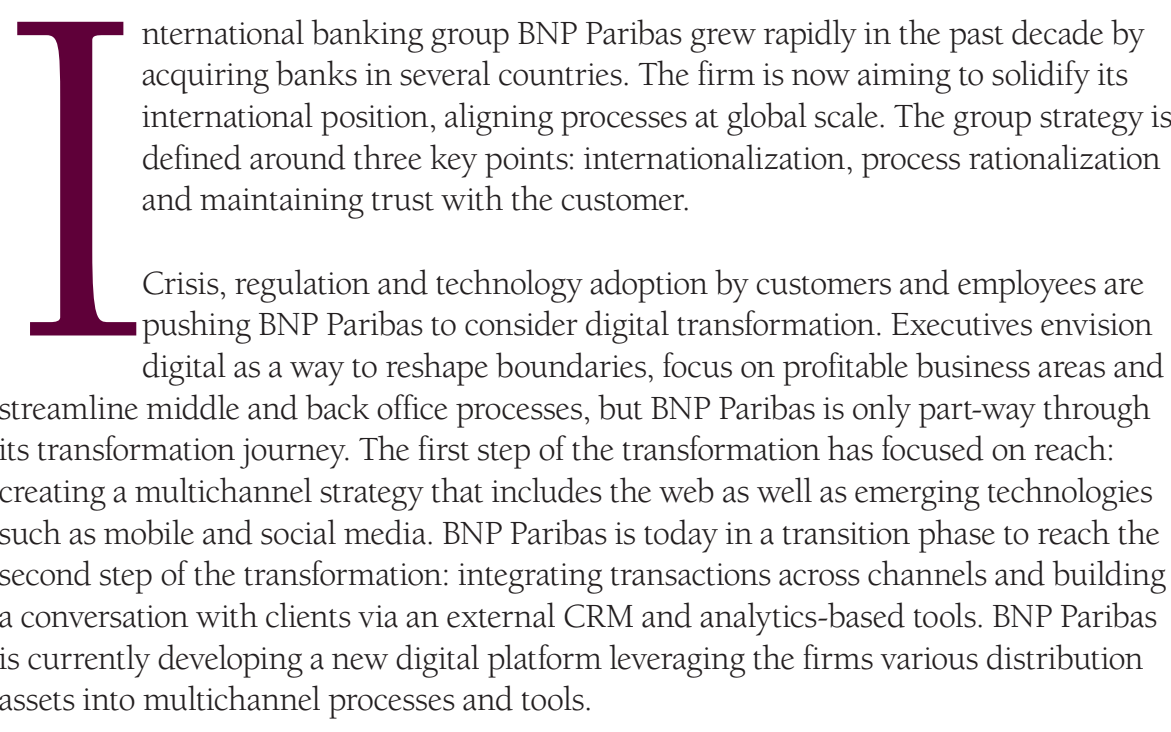

To improve customer experience, the firm launched multiple initiatives. Mobile apps provide a substitute to using the internet website. The firm increased customer touch points via social media, providing customer service on twitter and advertising on Facebook. In 2010, BNP Paribas launched a new branch model. This Concept Store will help develop new ways of interacting and selling products to the customer via new technologies and self-service areas. The firm's next step is to start a conversation with customers on social media. This will require building social-customer knowledge, via an external CRM, and increasing analytics capability. In 2011, BNP Paribas launched new mobile bank services in partnership with a telecom operator. This was more than a simple website substitution. It was a switch from traditional business model to a digital one, with a different cost and revenue structure.

From an operational process point of view, BNP Paribas is still at the beginning of its transformation journey. The firm is focusing on security improvements and cloud solutions, which executives see as key differentiators in the industry. The objective of process rationalization will be to have end-to-end digitized processes in an integrated platform, which could generate international economies of scale.

A group digital committee sponsored by general management elaborates the group digital strategy and encourages digital best practice sharing.

BNP Paribas' next digital objectives are to move into mobility, reach the next level of customer intimacy and develop a strong analytics strategy. Challenges on this road are to strengthen digital coordination at the group level and to balance digital and physical in the front-office perspective 
Case Study

\section{MOVING FROM SEPARATE TO INTEGRATED DIGITAL INITIATIVES IN AN APPAREL MANUFACTURER}

n apparel company has built its successful business by
combining good products with compelling branding and a set
of high-profile celebrity relationships. Its entrepreneurial spirit
has led to growth in product silos. The firm spent the past
ten years improving internal processes and its connections to
manufacturing partners.
The firm's entry into digital occurred in three successful
but disconnected areas. In customer experience, the firm

developed a customized products offering. Customers can go online or work with an advisor in a store to configure products in exactly the color schemes they wish, and then receive the products by mail. This process modified existing customer-facing and manufacturing capabilities to deliver a new customer experience. Separately, the company made forays into social media. It built communities around different categories of product, allowing customers to communicate with the company and each other via Facebook and Twitter. It also launched a few digital products meant to augment the lifestyle needs of customers, such as GPS devices for athletes.

In internal operations, the product design area moved from paper-based design processes to a fully digital process. Although some designers initially experienced trouble in the transition, the process has yielded important benefits. Shipping digital designs instead of physical prototypes between headquater and suppliers has cut the product development cycle time by months. It also allows the company to adjust designs much more easily in response to manufacturing issues or changes in customer demand. In addition, the firm can use digital designs to promote new products to retailers differently, gaining attention and orders much earlier and making retailer promotion easier than was possible with a physical design process.

The company has recently recognized the need to begin coordinating its digital efforts. It has created a digital division to deliver all digital efforts for the firm. This included recruiting key senior executives with experience in different aspects of digital business management, and building an innovation unit charged with identifying new customer and process approaches that cut across the firm's traditional silos. The digital division does more than enable and capture synergies between initiatives. It also opens up new possibilities for taking the firm from a product-focused to a customer-centric approach. 


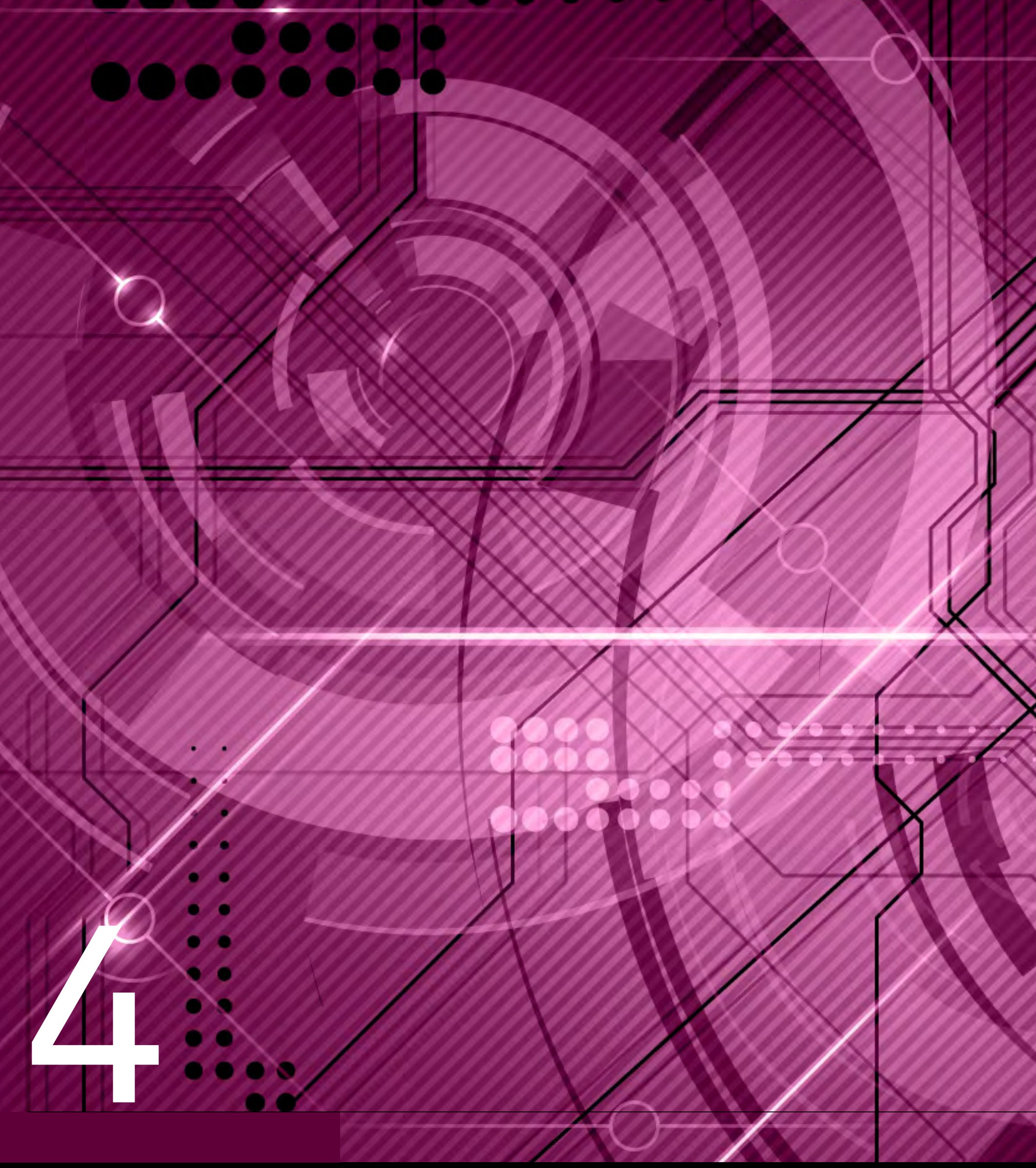

DIGITAL TECHNOLOGY USAGE 
The interviews asked how four digital technologies - analytics, mobile, social media, and embedded devices - are being used in the enterprise. All of the executives we interviewed were aware of these technologies, although few considered themselves experts. Most could describe how the company used the technology, or how it might or might not be important in the future.

Figure 6 shows how often firms in the study used each digital technology to improve customer experience and operational processes. Analytics, Mobile and Social Media are widespread in these large organizations, despite their relative newness to most industries.
Customer-experience applications of social media are more common than internal uses, reflecting awareness of the fact that consumers are rapidly adopting these technologies, and well as some skepticism about how effective they will be for internal collaboration. Analytics, too, is more common in customer experience applications, reflecting organizations' increasing efforts to target marketing activities and personalize customer experience better than in the past. Mobile is used widely in both domains.

Embedded devices are still an emerging technology. Less than onefourth of companies are using these

\section{Figure 6: Applications of digital technology in customer experience and operational process}

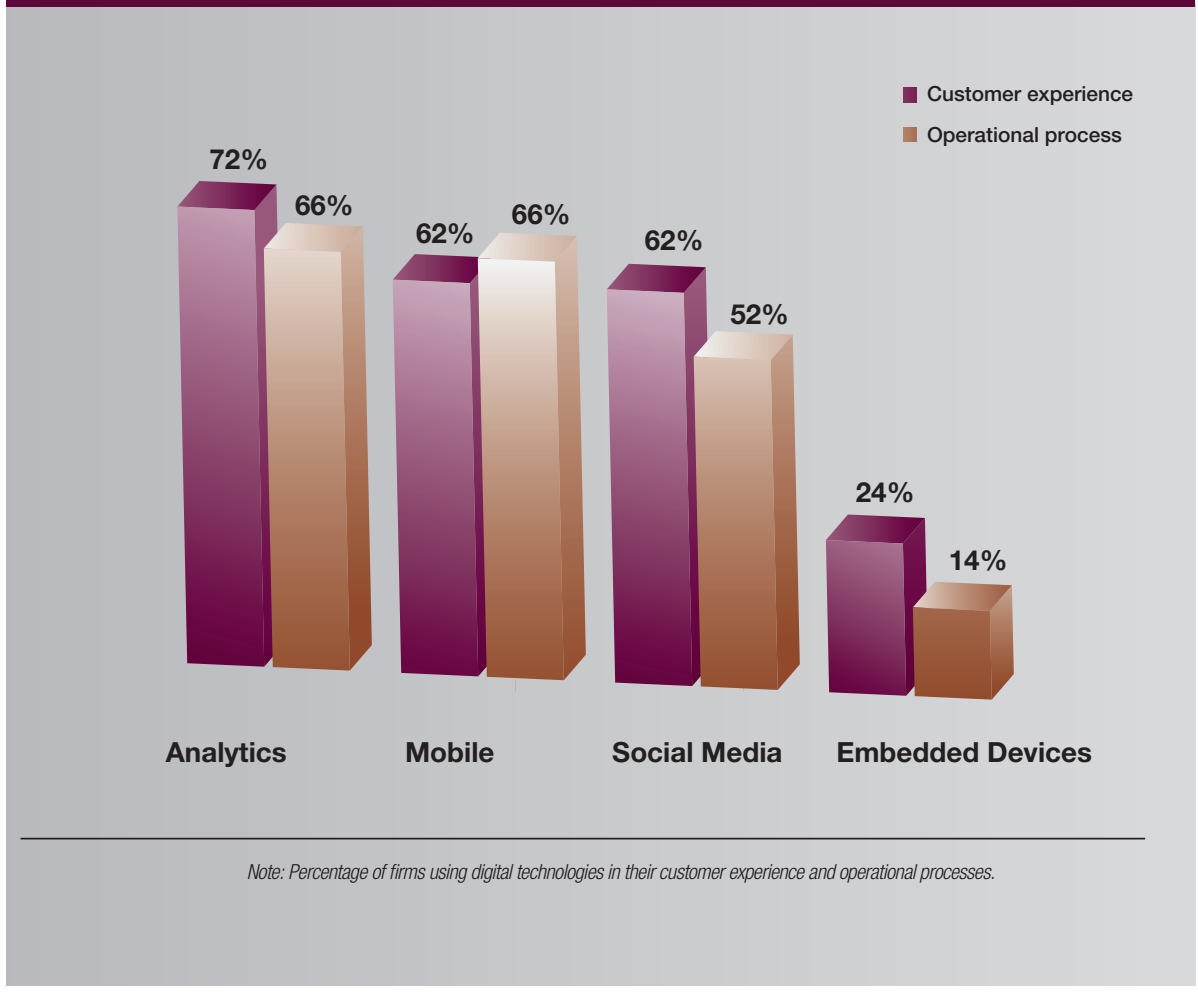


devices. Most executives commented that they did not yet see applications for the technologies. In the case of RFID, they mentioned the cost of the RFID tags or the difficulty of using them in busy, machine-filled spaces. Many executives did express interest in using embedded devices in the future.

Companies varied greatly in the ways they used the four technologies. We identified three levels of usage:

- Substitution is using new technology as an alternative or replacement for substantially the same function that the enterprise already performed with a different technology and process. Blackberries and iphones substitute for PCs in both internal operations (email) and customer experience (web access). Many employees use both methods depending on their needs at a point in time. Analytics, in the form of basic business intelligence tools and detailed reporting, substitutes for similar information sources available through other methods. Substitution is useful when it improves performance over, or is more convenient than, traditional methods.

- Extension is significantly improving performance or functionality of

\section{Figure 7: Applications of digital technologies in the enterprises we studied}

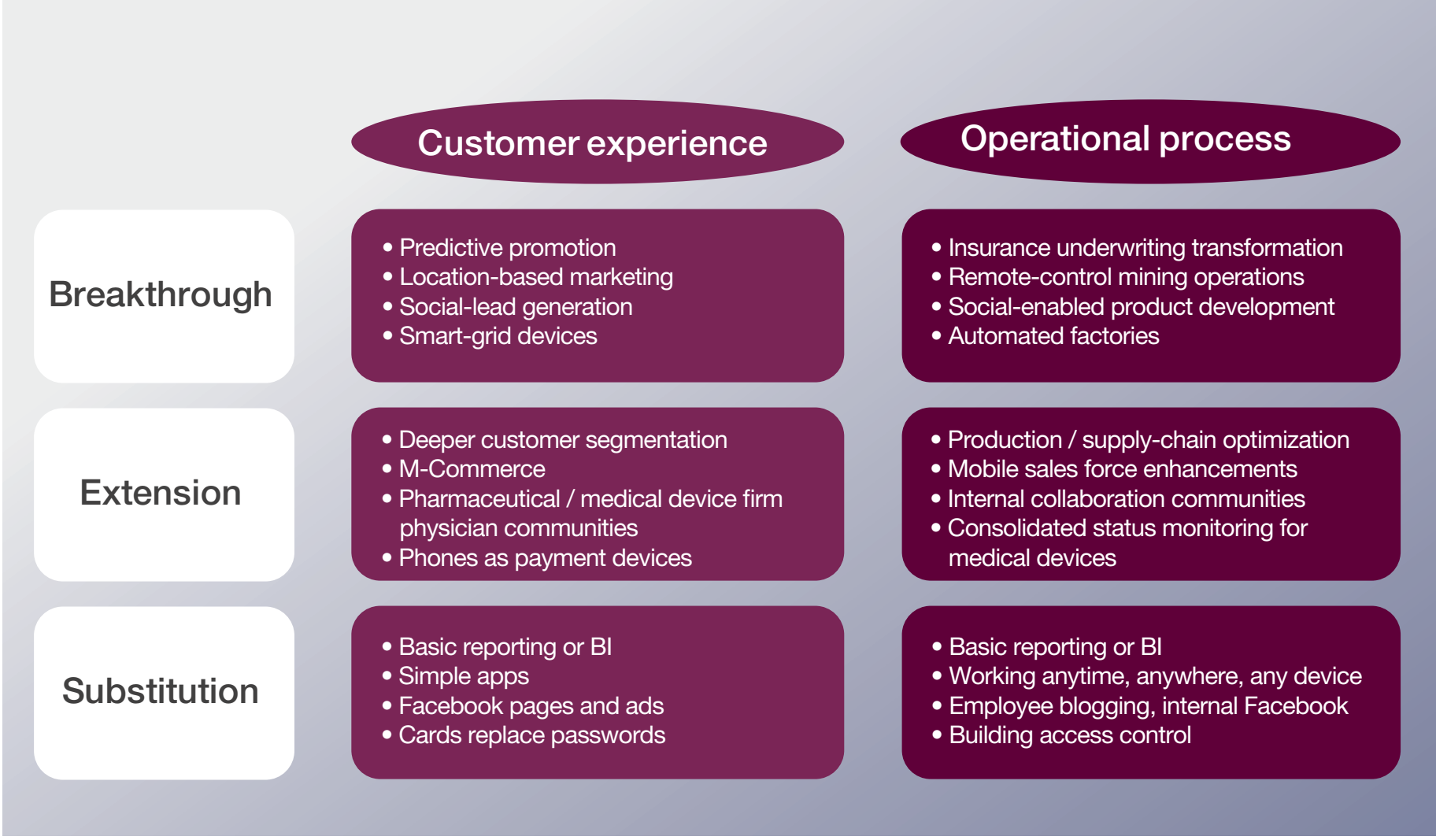


a process through technology. A power company and a paint company provide full information to field workers on mobile devices, eliminating the need for workers to spend time in the office at the start and end of shifts. A pharma company's social media physician community enables the company to learn about issues and opportunities by allowing doctors to talk with one another.

- Breakthrough is fundamentally redefining a process or function through technology. A paint company uses a combination of embedded devices and analytics to create fully-automated factories that deliver higher efficiency, quality and environmental protections than manually-tended factories. A hospitality firm uses predictive analytics to engage in location-based marketing with its customers.

Figure 7 shows examples of how companies are using the four digital technologies. Figures 8 and 9 show the extent to which each is being used to improve customer experience or internal operations. The most common usage of each technology is in substitution -- Mobile email replaces desktop email, Facebook pages and ads substitute for web. However, companies are making inroads in each technology. Roughly 30 percent of firms are engaging in extension or transformation with Mobile, Social, or Analytics. Although usage of embedded devices lags the other technologies, some companies are already extending or transforming their processes through these devices.

\section{Figure 8: Use of digital technologies to transform operational processes}

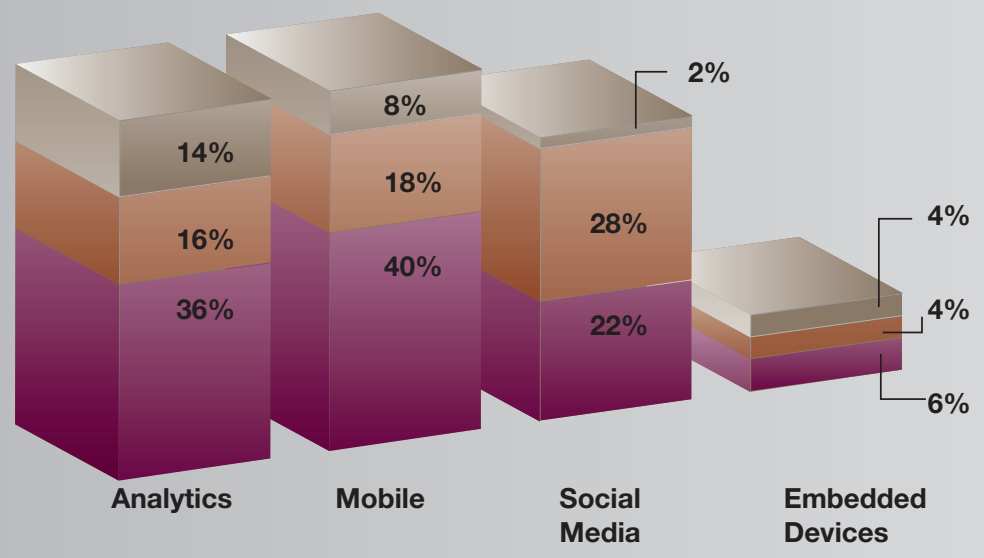

Substitution

Extension

Breakthrough

Note: Chart plots extent of transformation each firm is attaining in operational processes using a particular technology. Many companies had multiple applications of a technology. For each firm, only the most transformative usage is counted. 
These types of multi-technology solution are signs of a maturing approach to digital transformation in enterprises. Executives are beginning to envision possibilities that cross organizational and technological silos - letting the possibilities drive technology and organization rather than the reverse.

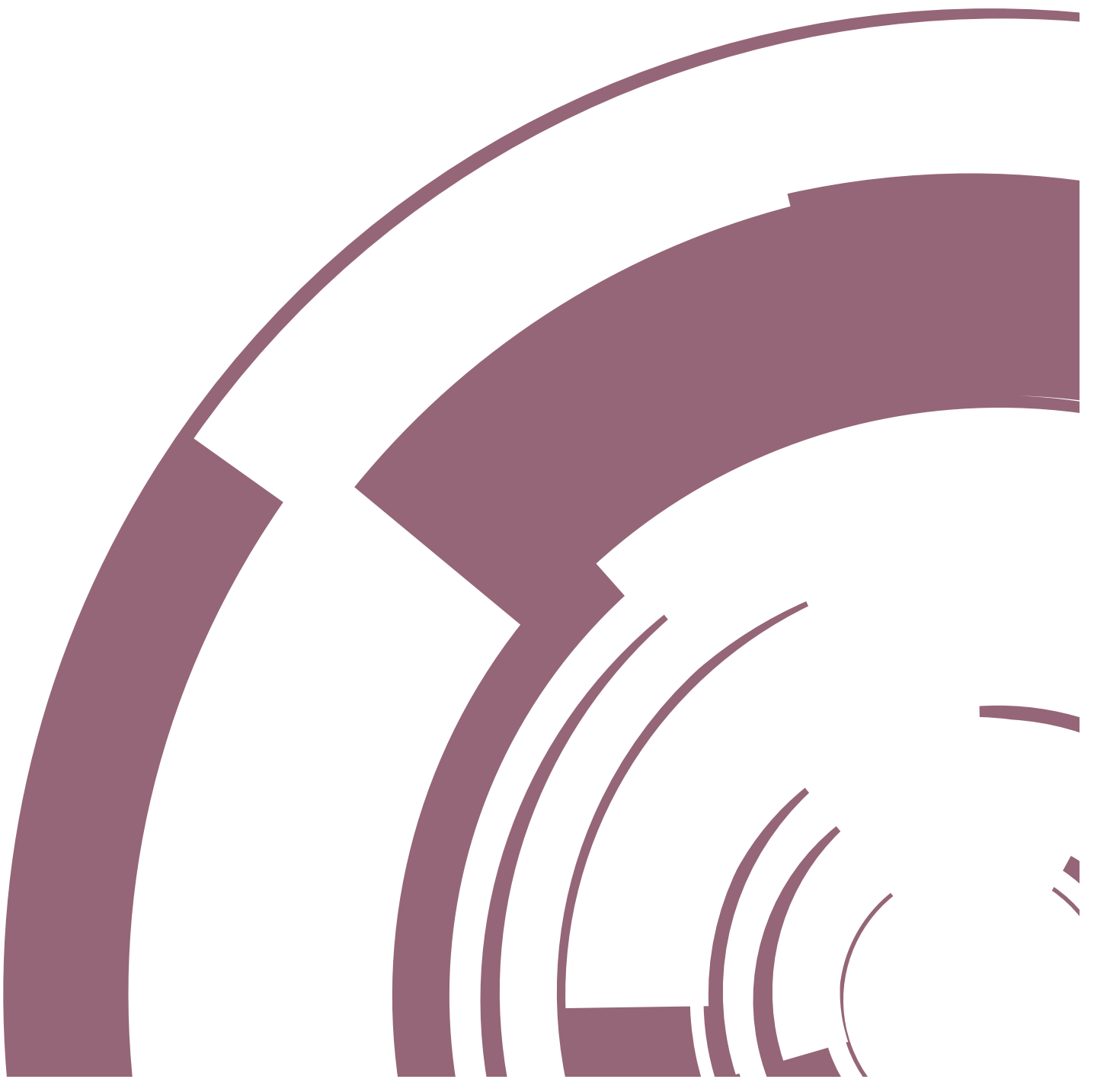




\section{ll|}

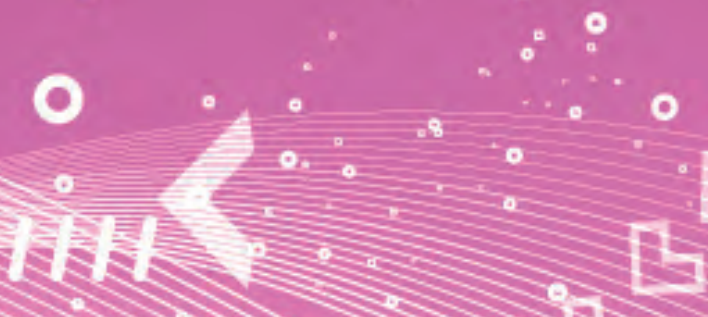
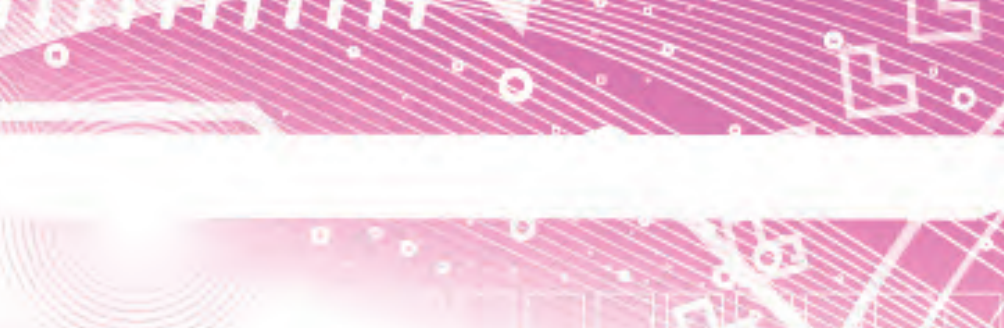

3

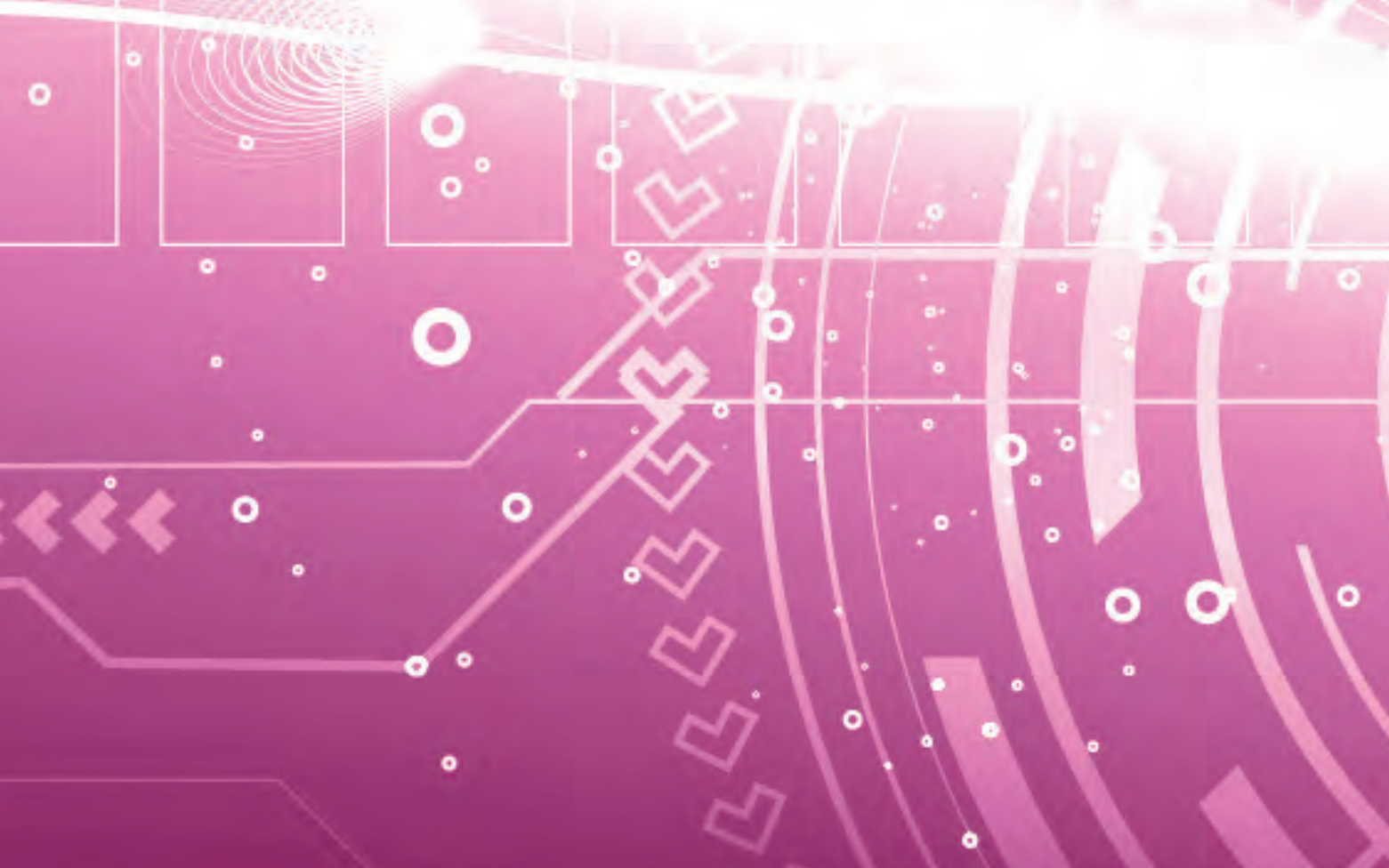

CHALLENGES ON THE ROAD TO TRANSFORMATION 
What is causing firms to have difficulty starting or benefiting from digital transformation? Challenges occur in all three elements of the transformation process: Initiation, Execution, and Coordination.

\subsection{Initiation challenges}

\section{Lack of impetus}

Impetus often starts at the very top of the firm. Executives are justifiably skeptical of the benefits of emerging technologies. The experience of e-commerce taught many executives that a fast follower approach can sometimes be lower risk than a pioneering approach. However, this skepticism can result in bureaucratic investment processes that prevent the firm from engaging in useful digitallyenabled experiments and business changes. A manufacturing executive stated: "We've always been fairly slow in adopting technology because we more or less have the ambition of not being first ...but we have become even slower at the same time that technology development has been speeding up. So, the gap has increased."

Company performance can also slow the move to transformation. If the company is not experiencing pain, the perceived risk of change may outweigh the potential benefits in the minds of many executives. A manufacturer said "At the end of the day, we don't want to stay on the edge of the latest trend. It's not really our business."

An other concern issue can be lack of awareness of the opportunities or threats of digital transformation. An executive in one of the world's largest financial companies said "When I made a presentation to the board, I was astonished that none of the senior executives were aware of TripAdvisor." Executives need not be aware of all changes in industries outside their own, but knowing major digital consumer services products such as Tripadvisor, Facebook, or eBay can be very useful fodder for envisioning how executives might change their own businesses.

\section{Regulation and reputation}

Many executives, especially in healthcare and financial services, are being careful about mobile and social technologies because of security and privacy concerns. In our sample, 47 percent of companies stated a high or medium concern over regulatory impacts on their potential digital initiatives. A lost or hacked mobile device can give outsiders access to confidential data on patients or customers. This has reputational repercussions well beyond any regulatory penalties. A financial services executive said "The last thing that any of us want to do is put the reputation that we built for more than 100 years on the line because someone lost a device." A medical executive had a similar concern: "We've done a good job of becoming a trusted partner to a lot of governments and customers. If we violate that trust, if we have one bad apple, you can throw the whole thing out the window very quickly."

Companies, especially in financial services, are taking a slow approach to social media for fear of regulatory sanction. For example, blogs, tweets and other messages are typically not reviewed as carefully as other corporate communications, but may be construed as advice by readers. A financial company executive said "We 
jumped into going ahead with Twitter and YouTube and Facebook without knowing what people are actually saying about us. Now we have started to monitor it."

While these regulatory concerns are real, they need not prevent companies from moving forward. Two financial services firms and one medical device firm are already successfully selling products and services with tablets instead of paper. Several firms have implemented tools to protect confidential information on phones and tablets or to wipe the devices in case they are lost. Nearly all of the regulated companies we interviewed are establishing policies for employee use of personally owned or mobile devices and for using social media. Many firms we interviewed are using Facebook and Twitter for brand building. Meanwhile, an insurance firm is helping agents build their presence on LinkedIn, and a medical device company plans to use Facebook as a radically new way to reach the specialists who use its products.

\section{Unclear business case}

Healthy skepticism or regulatory concerns are legitimate reasons to be careful when investigating new technologies. Certainly not all digital initiatives make sense for all companies (or for all employees in the company). One $\mathrm{CIO}$, discussing mobile-enabled process changes, said "At the end of the day, we're still looking at value and cost. For some folks, the most cost-effective technology is still the desktop." However, these reasons should not prevent companies from investing in potentially valuable experiments or transformations.
Executives in our sample used a set of four methods (see Figure 10) to justify investment in digital transformation initiatives. Some initiatives can be justified in the traditional way, through quantified economic business cases. Audio and videoconferencing technologies, for example, can be justified on travel cost savings or other efficiencies, as could a financial firm's move to a transformed office environment. Larger process transformations also can cite clear returns, such as when an apparel firm justified its move to digital design processes through cost savings and significantly shortened product development cycles.

However, other investments are truly bet-your-company, do-or-die propositions, in which the financial case is one of survival. Firms facing a burning platform often make large changes without a strict financial business case. A business directory service, realizing their paper-based model was declining rapidly, brought in a new CEO to lead a radical digital transformation. An airport authority invested heavily in digital transformation when governments stated that they would withdraw 60 percent of public funding within 5 years.

However, as with many innovations, digital transformation investments often have less clear business cases than these examples. Many companies considered their initial forays into Facebook or Twitter as simple experiments built with limited funding and risk to investigate the potential of the new media. Similarly, initial mobile apps or pilot experiments with mobile marketing have been scoped to limit cost and risk. These experiments are 


\section{Figure 10: How companies justify their digital transformation investments}

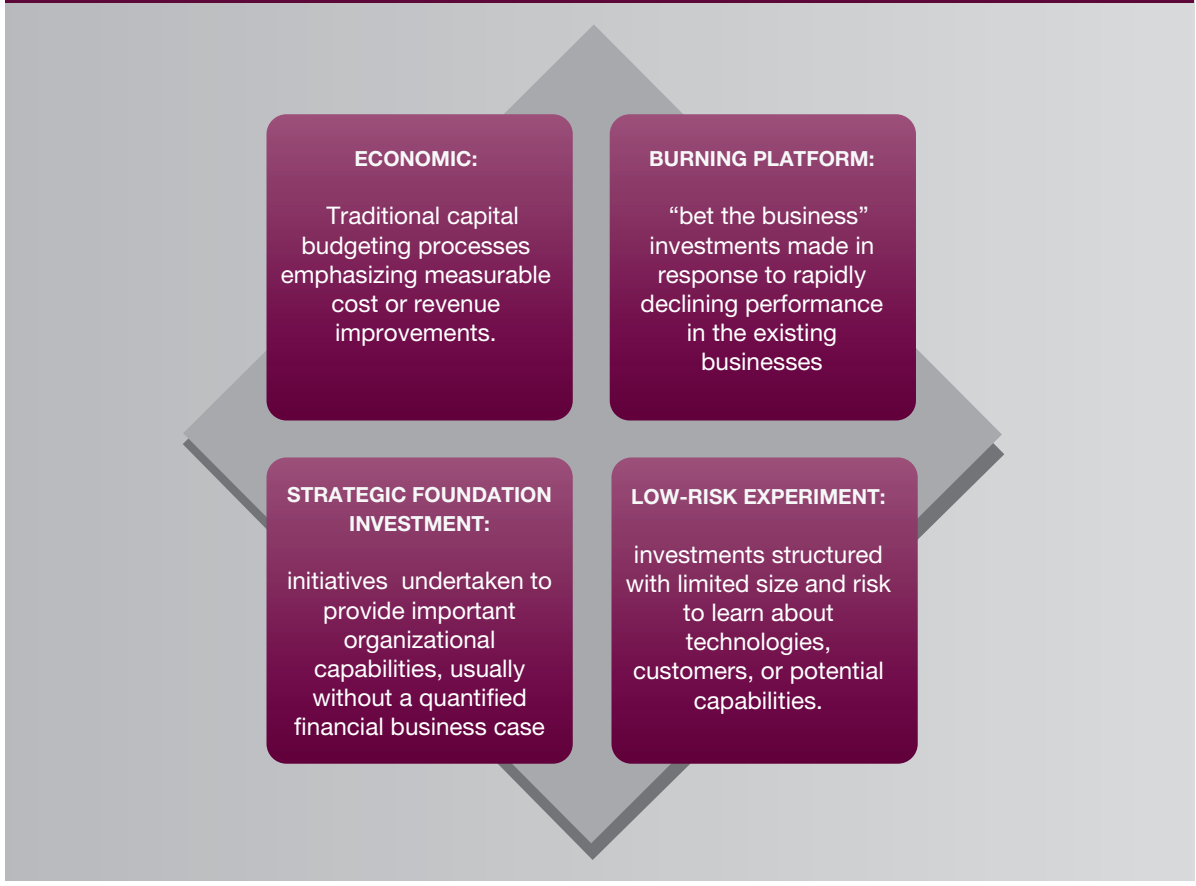

often funded "below the radar", using the spending authority available to a senior executive.

Other initiatives are funded as

strategic foundation investments that build infrastructure and capabilities to be used elsewhere. These investments rarely go through the rigor of developing a quantified business case, but rather are undertaken as strategic bets. Collaboration tools and internal knowledge bases, for example, were often justified as relatively low cost/ low risk infrastructure investments to smooth cooperation and synergies among a distributed workforce.

However, some foundation investments are larger. A manufacturing firm invested in building a shared digital division to provide capabilities and support to digital initiatives across the firm. Each new digital initiative would be funded on its own merits, but the initial investment to set up the shared service was seen as a strategic foundation investment. Similarly, several firms hired senior executives or content experts to get started in the analytics and social media spaces. The new leaders must financially justify their existence in the future, but the initial investment was considered as capability-building.

When enabling larger transformations, executives often use a combination of approaches over time. Analytics is an interesting example. Specific customer segmentation or predictive analytics efforts can often show a business case. Marketing experiments either generate higher sales or they don't. Insurance analytics either generate a better risk/return profile or they don't. However, firms often require 
an investment in infrastructure integrating data, buying tools or hiring expertise - before they can start to conduct more measurable analyticbased experiments. For a restaurant firm considering analytics, the return for hiring new skills and conducting initiatives was initially unclear, but senior executives believed it was a bet worth making to understand better sales trends and start to engage in dynamic localized pricing experiments.

Regardless of how an initial investment is justified, the interviews highlighted an important point. The real value of digital transformation comes not from the initial investment, but from continuously re-envisioning how capabilities can be extended with digital technology to increase revenue, cut costs or gain other benefits. Initial investments, made with or without a financial business case, become foundational capabilities on which additional investments can be made. When it first centralized order taking and implemented an ERP system, executives at the home improvement products company did not envision the many successful business model changes they would be able to add in the future. But, to their credit, they continuously envisioned what else they could do with their digitally-enhanced foundation, and earned higher and higher returns as a result.

\subsection{Execution challenges}

While a top-level impetus for transformation is important, it is often not enough. Interviewees cited three missing elements that threatened to prevent them from moving forward successfully (see Figure 11).

\section{Missing skills}

Analytic-based decision-making does not always come naturally to people accustomed to using "professional judgment" or other methods to make decisions. A grocery CFO lamented "We have all of the information in place, but we can't get those guys to change the way they work." Emerging technologies are another area where skills may be scarce internally, especially in areas such as mobility and social media.

Firms are filling gaps by hiring experts or working with vendors. Skills that are in short supply in one industry can sometimes be found in others. A restaurant executive said "We have been hiring analytics experts from other firms. We give them a chance to play a more senior role than they could in their previous employers, where their skills are more plentiful." A medical company executive said "We missed the boat on social media. We just hired a global CMO to take care of our social media strategy." Meanwhile, although the executive recognizes that smart embedded devices will be essential to the firm's future product strategy "We have nobody to look into that." A hospitality firm, aiming to limit long-term HR investment while experimenting with new technologies, is working with vendors who have the right skills and are willing to work for reduced rates to get a foot in the door. However, it is encountering knowledge integration issues as key skills are located in different vendors.

\section{Culture issues}

Cultural issues can also become a bottleneck for digital transformation. A major cultural issue is related to changes in jobs due to automation or information empowerment. A 


\section{Figure 11: Organizational gaps in digital transformation}

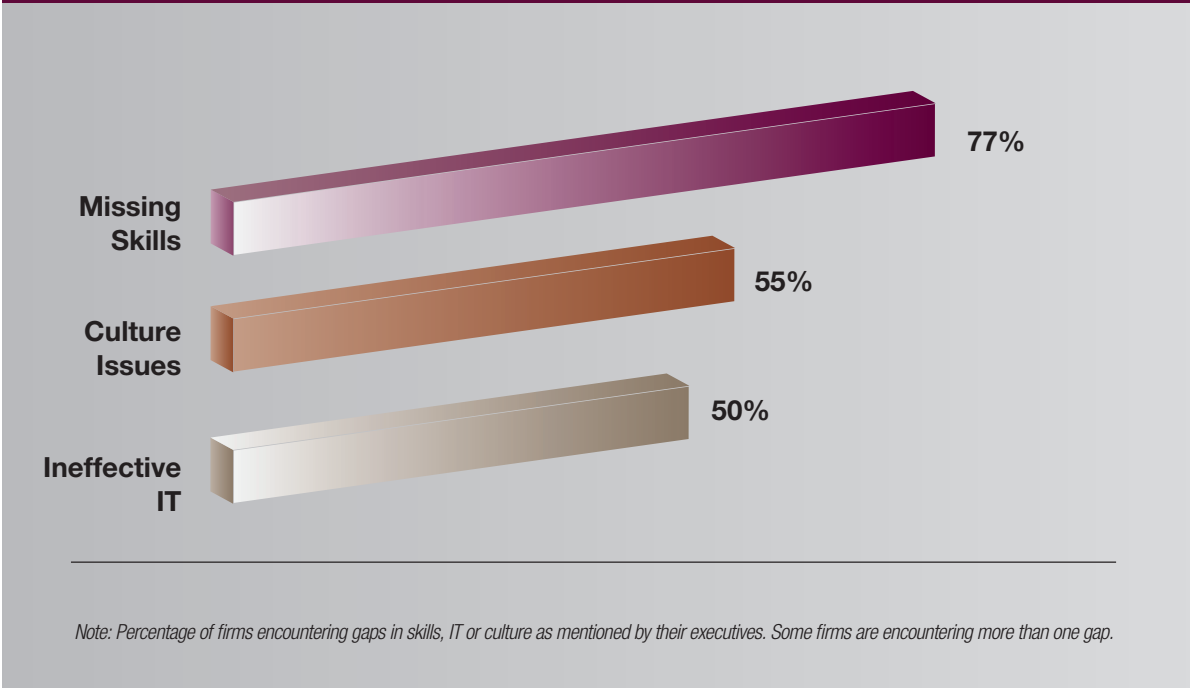

grocery executive mentioned that the firm's information-based benefits are reduced by managers who cannot think differently about how they make decisions. Other executives find that they must work to change the culture when empowering front-line workers. Executives shared that these workers, accustomed to taking orders from headquarters, needed help to adopt the idea of using new information capabilities to make autonomous decisions. Meanwhile, previouslyautonomous country managers in globalizing firms often require coaching to grow comfortable working in an environment of centralized shared services and standardized processes.

One lever for change is to hire new leaders. These people, while bringing new skills, also bring new vision that helps change the culture in their areas. A business directory company, apparel company, and restaurant firm have all benefited from this approach. However, whether leaders come from inside or outside, senior executives cite a strong vision and frequent communication as essential for driving culture change.

Labor relations issues are a stickier source of culture change issues. A business directory company, even in the midst of a successful digital transformation, continues to experience resistance from unions. In an airline firm, flight employees embrace digitally-enabled changes, but ground employees, fearing job cuts, are strongly resistant. These laborrelated culture issues can be overcome. Consider two airport operators from different countries. Both feel pressure to change from governments, airlines and customers. One company feels "stuck" in their environment, unable to move because of a culture that resists changes. The other operator overcame these cultural issues. It is now starting to partner with airlines to develop initiatives such as self-service drop-off, common check-in desk, and selling online airport-related (as opposed to airline-related) products to customers. 


\section{IT Difficulties}

Digital initiatives are built on a solid foundation of technology-enabled processes and data, as well as the analytics, solution delivery and relationship capabilities to create and extend that platform (see Figure 5). Information technology is a fundamental part of the firm's digital capabilities. However, many companies find their information technology infrastructures and capabilities severely lacking. According to the head of the disability business for a major insurer, "We have through the last 50 years proliferated our IT systems and applications. We don't retire systems. We just add on top of them, which creates a tremendous amount of expense and complexity." Data issues are felt especially keenly in marketing. One marketing executive, echoing the sentiment from many we interviewed, said "It is very difficult to get a good understanding of the customer when customer data is spread across so many systems."

Beyond technical issues in IT, relationship issues can be difficult. A history of poor business / IT relations is a difficult foundation for the collaborative work required in digital transformation. One business executive said "IT is a mess. Their costs are not acceptable. They propose things in 9-10 months where external firms could do them in 3-9 weeks. We started offshoring our IT, and now our IT guys are trying to change." A CIO described how he communicates about the possibilities of digital transformation, but none of his business executive peers (interviewed separately) seemed to find the discussions credible.
A strong IT/business relationship can be very helpful in transformation. The CIOs in a hospitality and medical devices firm have very strong relationships with other senior executives, enabling them to suggest and deliver important digital initiatives. A financial services firm has consolidated operations and IT under a single senior executive, enabling the firm to envision and execute process and technology changes in sync.

\subsection{Governance challenges}

Benefiting from transformation typically requires changes in processes or decision-making that span traditional organizational or functional structures. Transformation, like any major organizational change, requires top-down effort to help employees envision a different reality, and coordination to ensure the firm moves in the right direction.

\section{0 percent of the companies envision a radical digital transformation}

\section{Incremental vision}

While incremental investments can be effective starting places to build digital capability, the largest benefits of digital transformation come from truly transforming activities. This requires a more radical vision - one that offers a view of a different way of working, not just a faster or more efficient one. Unless senior executives establish a 
experiments in one site and then rapidly shares the learning across the company. Overly-limited visions can dramatically affect the value obtained from each digital transformation investment.

\section{Coordination issues}

Many firms fail to transform because of coordination difficulties across business units or processes. Units are able to make progress in their own areas, but are unable to influence practices in other units. The paint manufacturer could not have continued to benefit from transformation without top-down coordination of internal and customer-facing processes. Others are learning this lesson. A hospitality executive mentioned "We need to start rethinking our strategy. The approach of throwing was everything out there and thinking that something is going to stick is not the way to go."

An apparel firm, after conducting separate initiatives in design, marketing, and direct-to-consumer, is now investing in coordination. It recently created a digital division to ensure that all of its digital efforts marketing, production, design and even digitally-enhanced products - are being coordinated. The significant investment is expected to pay strong dividends from better customer experience, stronger knowledge of rapidly-changing customer preferences, and synergies between internal functions.

\section{A further concern arises from} coordination issues between new and traditional businesses or processes. This channel conflict is real, and can be very painful for managers in traditional units that lose when new businesses gain. It often must be addressed through an overarching vision. In a business directory firm, that vision was to have 80 percent of business online. In a credit firm, the digital division envisioned as small and complementary, so that it did not compete directly with the offline business. 


\section{Case Study}

\section{CREDIT FONCIER: EVOLVING AS A VALUE CHAIN ORCHESTRATOR IN MORTGAGES}

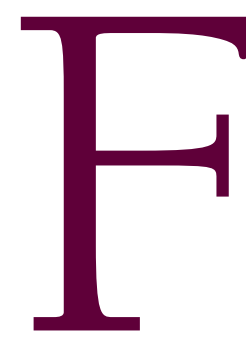

acing increasing pressure from competitors, regulatory changes, and customers, Credit Foncier decided to incorporate digital transformation into its business model. The firm, one of the top France's largest mortgage companies, aimed to augment its strong B2B capabilities by building a B2C experience that extended well beyond its traditional boundaries. It would orchestrate the entire customer experience of buying a property. This required envisioning a whole new way of operating, and then taking steps to experiment with offerings and educate both employees and customers.

A customer can go online or use a self-service kiosk in the concept "megastore" to learn how much she is qualified to borrow, or investigate what properties she is qualified to buy. The company can refer her to real estate brokers or provide financial products as part of the process. When the customer is ready to buy, she returns to the store or website to start the process of obtaining a loan. At any point, she can talk to an advisor who will help her in the process. The full experience is digitally enhanced, including color-coded maps, analytics-aided suggestions, and even a RFID-based key that the customer can use to save and retrieve her information in the store.

Internally, the entire process is integrated with analytics and a CRM system connecting elements of the B2C experience into the firm's other processes. The branch advisor can follow each customer through the process, offering help as needed. The company augments its existing assets by partnering with other firms from the long-standing web of business relationships that the firm has built in its B2B business. The company also hired new skills to augment its existing staff capabilities. The project, which was under consideration for 6 years, took 2 years to be implemented.

While the megastore concept has been successful, and the company is considering opening a new store in the near future, the company's focus remains on growing its successful B2B business. The vision for B2C is to augment existing B2B offerings and hedge against competitive pressure in the consumer mortgage space. As the concept matures, the firm will begin offering other products through the channel. 


\section{Case Study}

\section{AIRCO: DIGITAL TRANSFORMATION IN AN INTERNATIONAL AIRPORT}

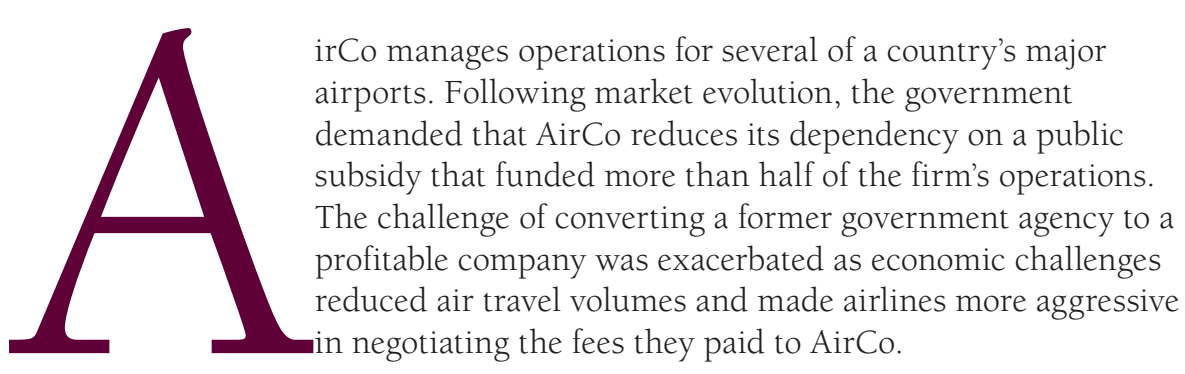

To gain new revenues, AirCo is transforming its business model. The firm's vision is twofold: to gain efficiencies by unifying processes across airports, and to focus on serving end customers instead of only the airlines. Implementing the transformation also requires engagement to help employees adopt more business-oriented perspectives. According to executives, however, the transformation benefits from two strategic assets: the firm's ownership of the airports (and thus control of how products and services are sold), and the employees' collaborative culture.

After unifying the management team, AirCo focused on rationalizing and integrating processes and IT infrastructure. It launched a digital transformation program owned by a $\mathrm{CIO}$ board that would coordinate and support the digital progress of the airports. The objective of the program is to allow the company airport to follow each customer as she moves through an airport, providing services wherever possible.

\section{Customer experience:}

The firm envisions increasing revenues from fliers to overcome diminishing revenues from airlines. It plans to take advantage of a major change in customers' behaviors: travelers are increasingly their own travel agents. In this world of self-service, the company believes it can help customers to reduce the stress of going to the airport, navigating traffic, checking in, and getting to the right gate through the use of a website or mobile apps. Customer apps already exist for the two biggest airports of the country, providing both the customer and the company with information about the trip. The firm supplements its digital services with information agents who roam the airport with tablets to support and guide travelers. AirCo will augment the apps with core business services like car rental, parking, and train tickets. The firm is also developing airline partnerships to provide airline tickets on the website. 


\section{Operational processes:}

The firm's biggest operational challenge is to have one digital strategy for all of its airports. It plans to provide every customer in every airport with the same services. The firm has started its platform consolidation and partnered with foreign airports to provide more information to travelers. It also made services such as drop-off or check-in available to the airlines, taking advantage of the interconnectivity of the firm's digital platform.

Digitization of operational processes is also improving the communication between employees. They are able to deliver a better service to the end customer. 75 percent of workers are already using mobile devices as they move through the airport.

The company does not have a social media policy, but has created guidelines. It encourages employees to be active on Facebook and Twitter. The firm has also launched a collaborative community platform to understand travelers' experiences better and to be able to adapt its services.

AirCo's digital transformation journey, currently underway, is seen as critical to helping the firm reach financial autonomy. Executives started with a clear vision that leveraged key strategic assets, hired a new $\mathrm{CIO}$, and then created governance mechanisms to improve the chances of success. These leaders also started early to build digital engagement with employees to change the firm's culture. Executives believe the firm is well positioned to implement the remaining elements of the digital transformation. 


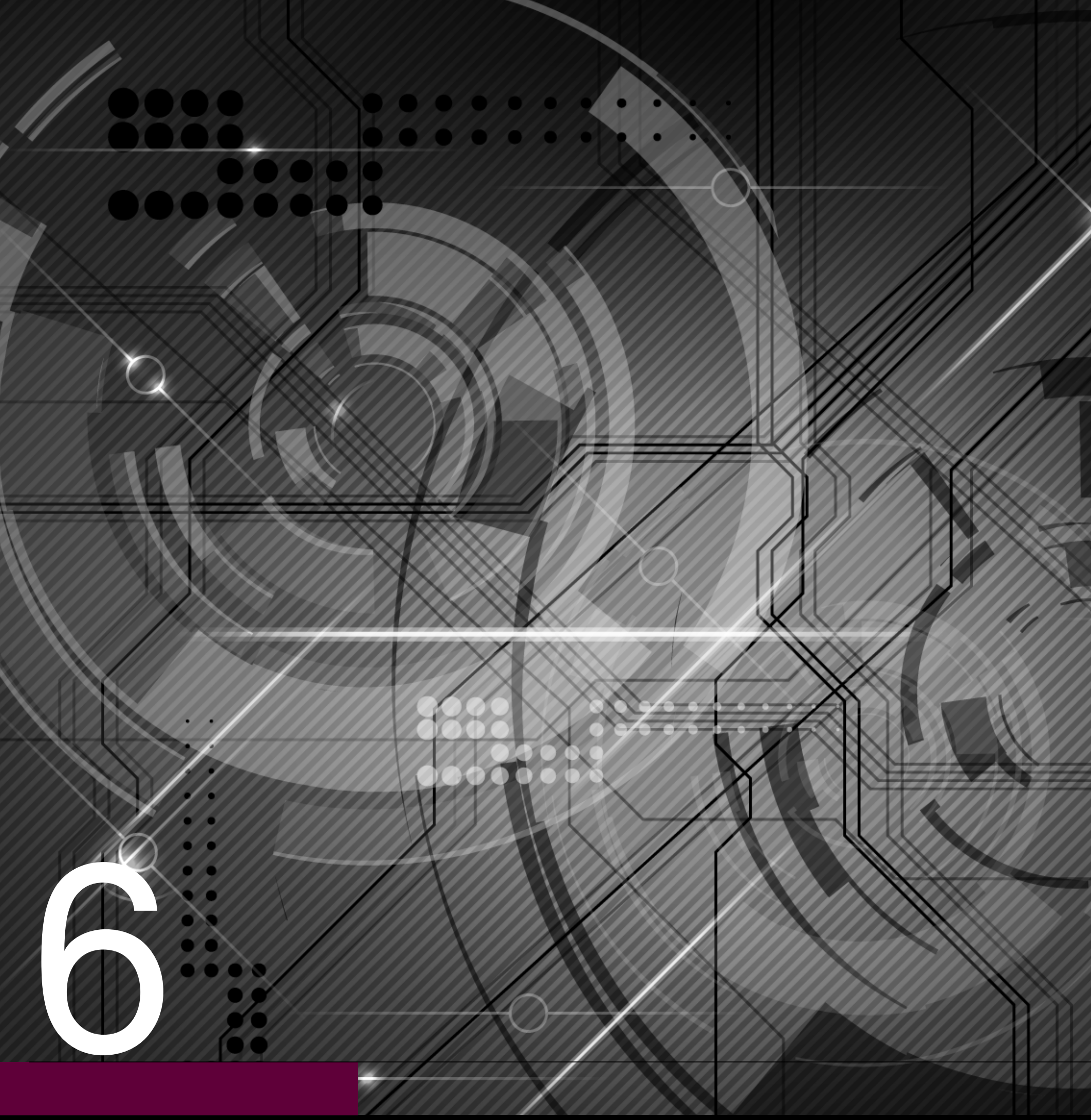

HOW CAN YOU MAKE YOUR DIGITAL JOURNEY SUCCESSFUL? 
Successful digital transformations in our study used a common set of elements (see Figure 12). Each is a lever executives can use to initiate and drive digital transformation in their organizations. Leaders diagnose the potential value of existing corporate assets and build a transformative vision for the future. Then, they invest in skills and initiatives to make the vision a reality. Fundamental to the transformation is effective communication and governance to ensure that the firm is moving in the right direction.
These elements work together in an iterative approach - constantly communicating and listening to re-envision and further implement new types of digital transformation. Senior executives drive digital transformation through an iterative three-step process:

1. Envision the digital future for your firm.

2. Invest in digital initiatives and skills.

3. Lead the change from the top.

\section{Figure 12: Digital transformation framework}

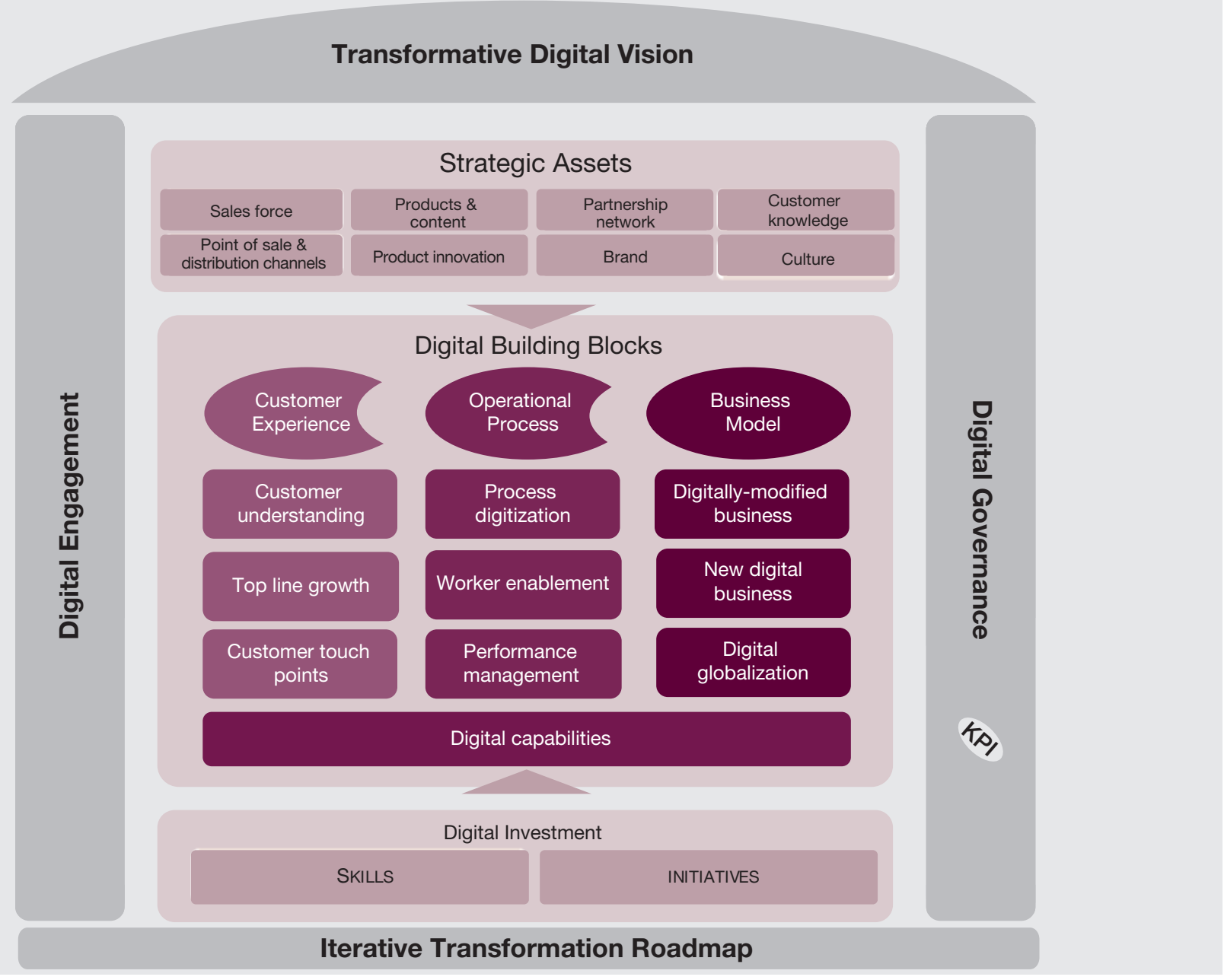




\subsection{Envision the digital future for your firm}

Many digital transformation initiatives fail to capture all of the value available to them because their vision is not transformative. Only the top of the company can create a compelling vision of the future and communicate it throughout the organization. Successful digital transformation does not occur bottom up. The true value of transformation often comes from seeing value across silos and then helping everyone else see that value.

\section{Identify and diagnose strategic assets}

Large companies survive major transitions not by radically replacing the old with the new, but rather by transforming some of their existing resources and competencies for the new environment. While this sometimes involves changing leadership or replacing assets, it usually involves reassigning or adapting assets and realigning or re-motivating employees. However, it also requires understanding when traditional assets and sources of advantage no longer provide value.

Digital transformation is the same. Your company will thrive in digital transformation not by doing something completely new, but by taking advantage of your powerful capabilities to gain advantage through digitization. But that requires thoughtful diagnosis: what assets will be useful in a digitally transformed world?
Interviewees, without being asked, often identified important assets that could help or hinder their transformations. These strategic assets include:

- Sales force: A strong source of customer loyalty is the set of relationships cultivated by a strong sales force. Digital initiatives can threaten to disintermediate salespeople, leading to channel conflict. However, digital transformation can also be used to enhance those relationships, such as a logistics firm that can use demand data in one part of the world to provide customers in another part of the world with forecasts of trends that will hit them soon. In addition to sales people, front line employees often are an important face to the customer, as well as having important knowledge about how business is done.

\section{- Point of sale and distribution} channels: Stores are often a strong source of location-based advantage, even in a digital world. In other cases, firms with a strong warehouse and supply chain capabilities are able to use their distribution assets to disrupt the advantage of local competitors.

- Products and content: Media firms find their content is a strong asset that can be reused in digital environments. Product companies often find they can build new digital business around strong products. An electronics manufacturer is building energy management solutions around its highly successful devices for the commercial buildings 
market, and a manufacturer of expensive long-lived transportation products is building services that digitally diagnose and help to maintain those devices.

- Product innovation: A high-tech materials firm has engineering capabilities that few other firms can match in its niche markets. It has the opportunity to use digital transformation to connect its engineers more closely with global manufacturers. An apparel firm has world-class fashion design talent. It uses digital technology to connect designers closer to manufacturers, speeding the design-to-market cycle time while enabling designers to change designs at will.

\section{- Partnership network: Strong} partnerships can be a key lever for transformation. Networks of exclusive or trusted relationships can enable firms to combine different expertise and deliver powerful new operating models. A mortgage company, in switching from a single value chain link to a value chain orchestrator, benefited greatly from its partnership assets. Partners could have refused the idea out of fear of direct competition, but they cooperated in the project because of years of working together on many financial products and transactions.

- Brand: Companies with a strong brand are able to leverage it in related offerings. Through mobile web, social media, new digital businesses and other digital initiatives these companies can extend and strengthen their brands, building additional points of contact with customers.
- Customer knowledge: Across the years, companies had gathered more and more knowledge about customers. Today, some are reaching a point where they can start envisioning the next step, monetizing this relationship to launch new products, enhance customer relationships or augment sales via customer-segmentation.

- Culture: Some firms are able to use culture as a powerful asset. Executives in a manufacturing firm found that the company's historically entrepreneurial culture made digital transformation easier. Employees were willing to embrace operational changes and strategic partnerships as part of the new vision. An airport authority noted that the nation's collegial culture made it easier to engage in transformation. Meanwhile, another airport authority found that its unionized culture restricted change.

\section{Create a transformative vision}

Successful digital transformation comes from envisioning new ways that digital technology improves performance and customer satisfaction, not just trying to find a use for the new technologies. As we described earlier, many companies that are doing experiments in mobile marketing, social media, or analytics find that they can be quickly stymied by organizational boundaries or by culture issues. Often the problems arise through vision focused on technology rather than different ways of operating. More often they are limited by a vision that is incremental instead of transformative. 
Start with an overarching vision of the what, not the how: "customer experience transformation" not "mobile marketing" or "social media." Then support the message through consistent communication. Each specific element of the transformation can then be placed in a context of the broader vision. This will also highlight when issues such as a single customer profile or a coordinated messaging approach may be important in reaching the vision.

Our interviews showed visions that were internally focused, externally focused, or bridging the two. Some visions were focused on specific business units, while others extended across the enterprise. Another element of the vision is the relationship between new and existing businesses. Companies in the study had visions that included launching new businesses, digitally improving existing businesses, or creating an overarching vision for a transformed company.

\subsection{Invest in digital initiatives and skills}

Transformation does not happen without investment. Digital transformation is no different. The investment may be large, and the

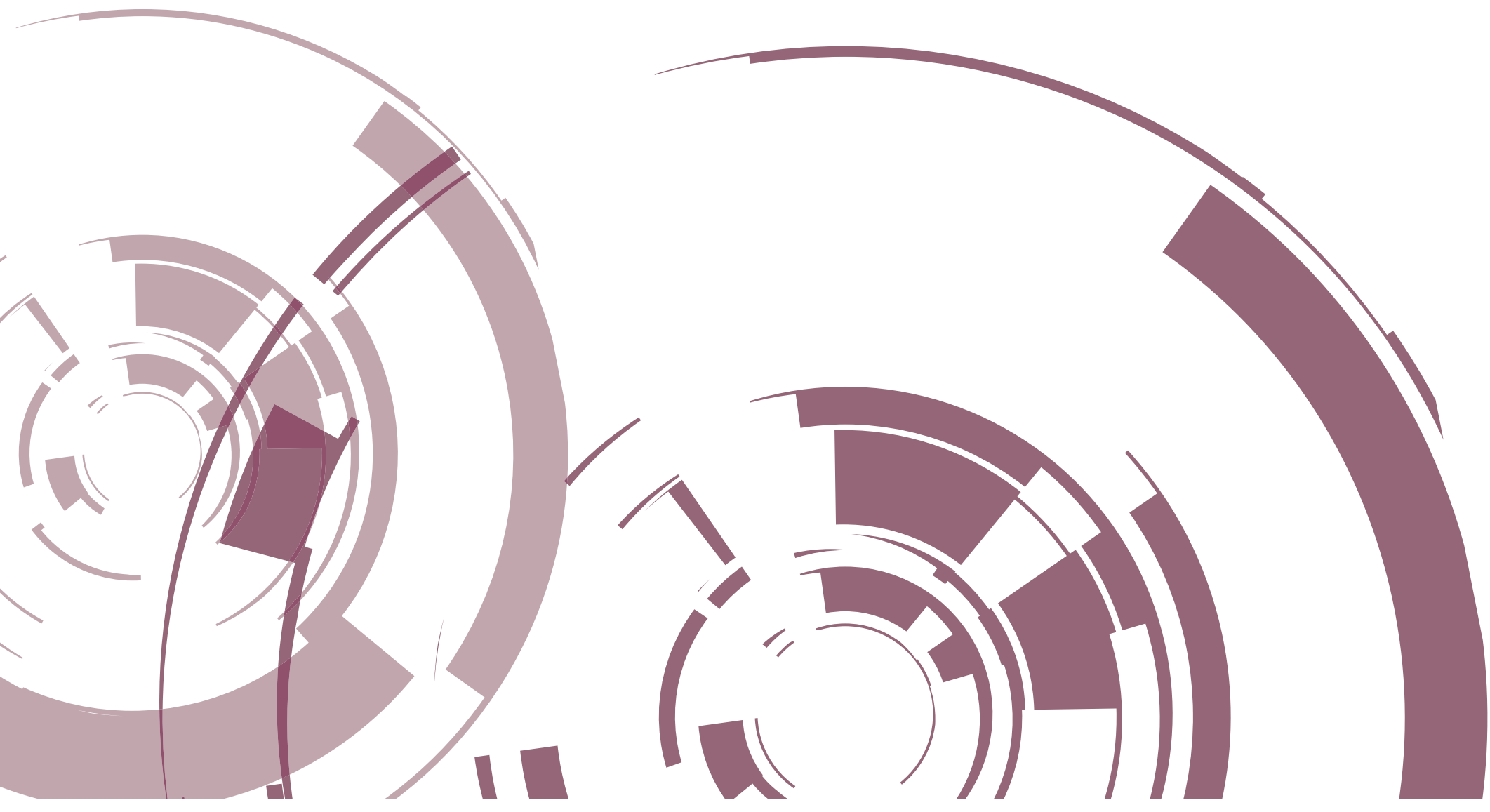


business case may not be completely clear. It may be a series of lowrisk experiments that lead to a larger investment. It often becomes a strategic bet that only senior executives can make. As with any investment, digital transformation requires understanding the need for investment, managing risk, and making the changes necessary to capitalize on the change. In addition, there is often tremendous value to be gained from making the most of investments you have already made.

\section{Find the right skills}

Respondents nearly universally described the difficulty of finding the right skills to manage new initiatives or to change work methods. While some were able to reassign or retrain existing employees for the new vision, most found it necessary to acquire skills from outside.

Find good vendors for technical skills, but coordinate them. When investigating a new technology, it is often easier to hire vendors than to hire people. Companies can hire vendors for an emerging technology experiment, and then easily end the contract at the end of the experiment. However, coordination is still necessary or knowledge will remain stuck in vendor silos.

Hire some stars. Analytics skills are becoming more plentiful, but are not distributed evenly across firms. One firm hired good analytics leaders from firms where these skills were more plentiful. The new people, who were skilled but experiencing limited upward growth in their firms, were able hit the ground running. The new hires created initial analytics capabilities quickly while helping existing employees understand what it means to work in an analytics-driven environment. An apparel company hired two senior people who were already well-versed in social media marketing and online commerce, asking them to help the firm build skills in those areas.

While outsiders can be helpful to jumpstart the change, companies also found insiders very helpful. In particular, it was very useful to put senior insiders in roles that required coordinating across units and changing cultures. Meanwhile, other firms found that they could move junior level fastrisers into roles where their energy and resilience was essential for success. The hospitality firm moved two relatively young managers into roles in mobile marketing and digital innovation. These two people oversaw rapid experimentation in their areas using very small staffs and a set of vendors. Both reported to a much more senior executive who could manage coordination across their units and with the rest of the firm.

\section{Invest in initiatives that advance the vision}

Very few examples of successful digital transformation were fully planned in advance. In many cases, executives built basic capabilities for one need, and then incrementally added capabilities. A set of relatively incremental changes added up to a radical transformation. A medical device firm implemented relatively incremental digital changes to enable knowledge sharing and videoconferencing, but then used them to radically transform its strategymaking processes. An apparel firm 
conducted digital transformation initiatives in product design and in marketing, and is now considering initiatives to link the two.

In other cases, radical changes were just the start of even more radical changes later. Executives envisioned a radical transformation of the company, invested in key initiatives, and then opportunistically made additional changes that advanced the vision. In addition to the home improvement products and directory services companies, a financial services firm is an example. Radically changing its headquarters design caused people to rethink the way they work. Instead of working in defined offices in headquarters, employees began to envision their work happening independently of location - moving desks as needed, working on mobile devices, collaborating virtually, or working from home. Then it was only a minor step to improve the way the firm collaborated globally.

As a leader, build a roadmap for your initial changes. Make it clear how the initial roadmap connects to your broader vision for the company. But then be open to ideas that will build on that foundation for even more change later on. It requires judgment, but also strategic bets. Not every idea will be a winner. By promoting a strong vision of a transformed organization, you put in place a process through which smart people throughout the company can help you to implement and extend that vision in the future. But then you must be open to hearing and sponsoring those extensions.

\subsection{Lead the change from the top}

Top-level vision rarely translates to local-level action unless reinforced through top-down communication and governance. Consistent engagement, backed with appropriate coordination, KPIs, and incentives, make the difficult process of transformation possible.

\section{Engage the organization}

As in most business transformation, the role of communication is paramount in effecting change and reducing organizational resistance. People can be a much bigger obstacle to successful digital transformation than any complex technology. Successful companies in our study recognized this by communicating early their digital vision and using technology to mobilize their workforce. There are two main characteristics of communication in a digital world that are making mobilizing the workforce easier and more impactful than traditional methods:

- Moving away from "cascading" information through traditional hierarchical channels toward enterprise-wide large scale communication. On top of traditional tools such as e-mail, new possibilities have been opened up with webcasts, internal social networks or PC-based video conferencing.

- Moving away from a oneway "broadcasting" model of communication toward offering people in the organization the opportunity to engage in a real 
dialogue around the transformation. Wikis, discussion forums, blogs and so on are easy tools to support such conversations.

Enterprise 2.0 tools are powerful tools to help mobilize and align the workforce towards digital objectives. Most organizations have already implemented some of these technologies but are not exploiting them to the full in their communication efforts. Successful organizations have not only created awareness through communication, but have increased the level of transparency and trust through this open dialogue.

\section{Establish digital governance}

Bottom-up digital transformation is rarely successful. With the right vision and leadership, opportunities from digital transformation build on each other to create new ways of working that would not have been possible earlier. However, they require
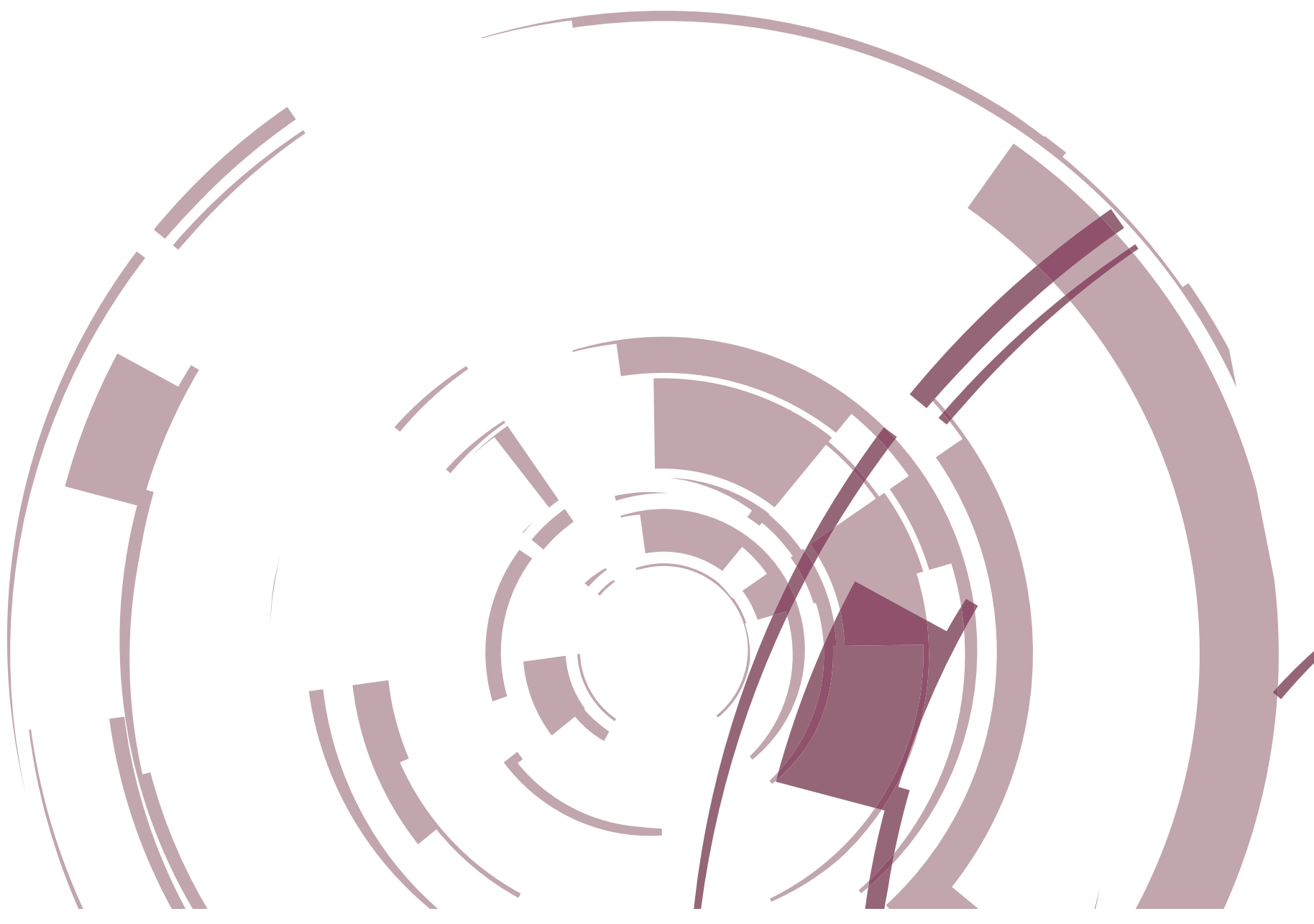
top-down ambition setting to put the organization in motion, coordination to turn disconnected or poorlyconsidered digital investments into true platforms for transformation and monitoring to ensure that progress is being measured and managed.

Ambition setting: Once an organization has defined its transformative vision, leaders must translate that vision into a set of ambitions and targets that signal to executives and employees "what good looks like." In our study, some of the companies most advanced in their digital transformation had established a clear set of ambitious KPIs to drive the desired results.
One executive stated "There's an intense focus on the transition to digital because, from the very top, the CEO has mandated that 20 percent of our sales will be digital within 5 years. To achieve that we will have to change the culture and the way the organization operates." In a financial company, the CMO stated "Unfortunately, digital transformation was not a priority of the board a year ago. We had 2-3 percent of digital revenue. Now this has changed and we have set a challenging target of 15 percent for all divisions." Ambitions need not be only about revenue changes. A home improvement product firm sets targets related to health, safety and environment, as well as to scrap reductions in plants.

\section{Figure 13: Coordination models for digital transformation}

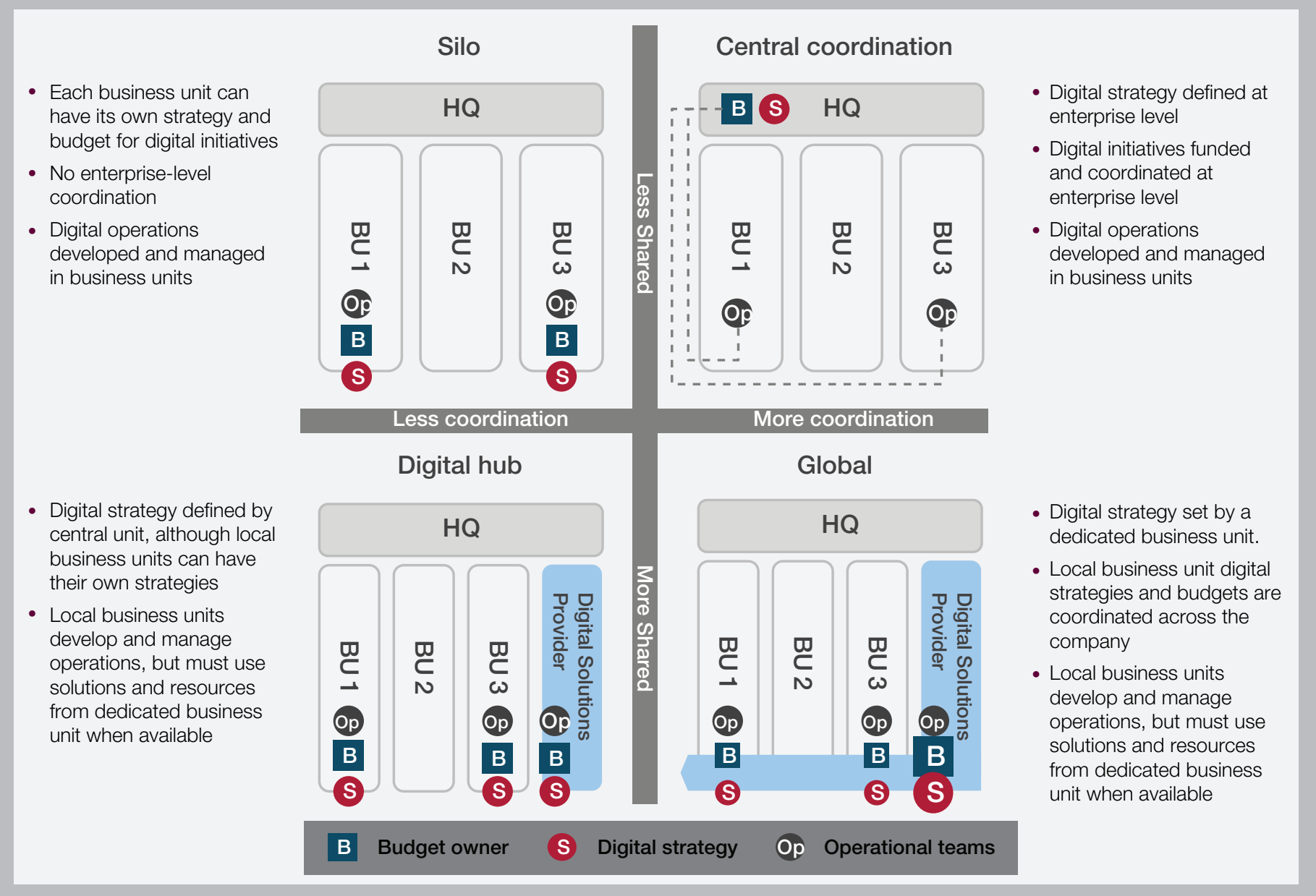


Coordinating mechanisms: Successful companies in our study had strong leadership for digital initiatives. Most had to adjust their organizations to accommodate the new digital ambitions. As the CEO of a digital business unit explained "We said we'd find the balance between central and de-central by creating one central role for coordinating the digital strategy. We appointed local Chief Digital Officers CDO in local business units reporting to both the CEO of the business units and a CEO of an overall digital business unit. In one of our business units we moved the CEO to the position of Chief Digital Officer. It was a promotion. So the Chief Digital Officer CDO role is more a business role than a technology role."

We observed in our study that not all the companies use the same structure. We found four models for coordinating digital transformation (see Figure 13). Figure 14 shows that central coordination is the most commonly used model in our study, but all models are relatively common. We found no single model that clearly outperforms others. However, we do see clearly that companies lacking effective digital coordination do not get the value possible from their digital transformation initiatives.

Monitoring progress through KPIs: Once the ambition is clearly stated and the organization structure is aligned, leaders need to ensure that a transparent set of metrics and KPIs are in place to constantly monitor and review progress towards that ambition. As an executive stated "To ensure alignment with our digital strategy, we monitor our progress through a transparent scorecard where everyone can see a quantified assessment of our progress and take actions against it."

\section{Figure 14: Usage of digital coordination models in the study}

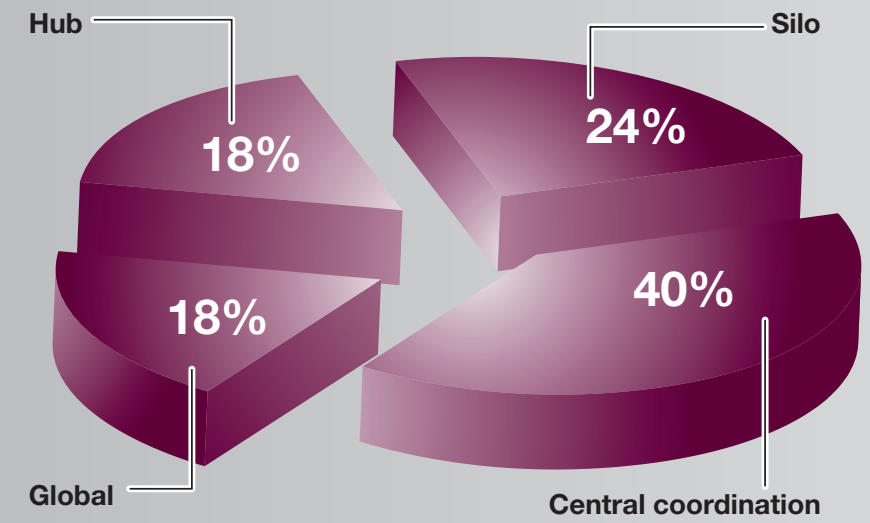




\section{Case Study}

\section{PRISA: DRIVING DIGITAL TRANSFORMATION ACROSS A GLOBAL COMPANY}

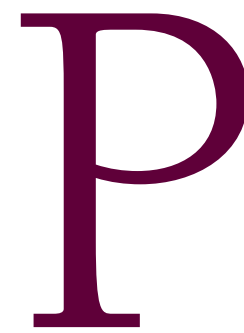

RISA is a Spanish-language-based global media organization with businesses spanning television, press, radio and educational publishing. As with many media organizations, PRISA was faced with a rapid transition to digital in most of its core businesses, and a move away from more traditional media. According to a senior executive, "We realized early that if we don't transform the way we do business, we're going to die. It's not about changing the way we do technology but changing the way we do business." The digital transformation was mandated from the top of the organization. The CEO, in laying out his vision of the future, demanded that the firm substantially grow its digital business from less than 1 percent to 20 percent of its sales within a span of a few years.

The CEO instituted a radical change for the highly decentralized organization by creating a centralized digital unit to coordinate and assist in building digital businesses. The appointment of a global Chief Digital Officer from outside the organization, who reports directly to the CEO of the Group, was a major signal. Senior executives were appointed to the position of Chief Digital Officer in each division, coordinating with the central digital unit. The Chief Digital Officer of each division was responsible for the implementation and coordination of the digital transformation of their division. This was a very senior role that had not existed before. For example,

The company's digital transformation was designed around four key pillars:

1. Creating a federated digital organization

2. Creating the next generation digital distribution and monetization platform to ensure that all of their global assets could be shared effectively across businesses, and new products could be easily created

3. Injecting new digital skills and experience from outside the company

4. Encouraging digital experimentation (e.g. social media strategy) and innovation (e.g. crowd sourced textbooks) in the business lines.

The company monitors the progress of its digital transformation through clear and transparent performance management scorecards. The digital unit is simultaneously driving innovation (an investment model), sales strategy (a P\&L model) and service delivery (shared service model). 
Through digital, PRISA faces cannibalization of its traditional business. The firm still has profitable traditional activities and there is a temptation to focus on retaining this current business. But the transition is being reinforced in communication from the top of the organization. For example the top management at PRISA TV talks about the evolving interactive landscape of digital video technologies, not just about the traditional set-top-box model of pay TV. Education is also moving fast. "It's not only taking a book and making a textbook that's digital. It's reinventing education. It's defending the old business through the power of digital opportunities."

PRISA is engaged in a strategic project with technology partners "to be able to deliver any type of content anytime, anywhere, any device." This digital platform will be fully centralized and support information sharing in the group: "It will enable them to get a South African interview of Christiona Ronaldo for El Pais local, and to publish it in Spain. Of course, the system will preserve some exclusive content for a certain time." With this digital platform, "PRISA will be able to offer a global platform to its customers with one identity -- fifty million customers' credit cards -- and start giving them a consequent ecosystem. No other media companies have yet done that."

For Mobile, the company faces market issues as executives believe the advertising market is not well educated on the new cost structures. PRISA launched some initiatives like an app for El Pais that was recognized as the world's \#1 media-related iPad apps in April 2011 (see http://mcpheters.com/2011/04/05/ imonitor $^{\mathrm{TM}}$-releases-list-of-best-publication-apps/).

In Social Media, the firm is doing some experiments. PRISA has been on Twitter for a couple of years and does some advertising on Facebook. The company has integrated its digital products more tightly with social media and intends to use social media information to drive content consumption. The company views social media, mobile, and video to be key drivers for future growth.

PRISA also envisions several new business opportunities "So if you're watching, for example, a soccer game of Real Madrid and you see players running around, you're going to have technology that understands the numbers on the back of the shirts, and it can go up to the database, and then they can say 'give me the stats on these players' and pull them up on the right side of screen. That's an interactive content analytics play. And maybe you can tweet about it right there."

PRISA's transformation challenges have mainly been around cultural resistance, the differing speeds of transition in each business unit, digital skills building, and allowing for experimentation while keeping the integrity of the unified strategy. The firm's top three challenges for the future are breaking risk aversion, streamlining the decision making process, and executing technology on pace to support all of the needed changes. Digital will also enable PRISA to expand to other European countries and to deepen its Latin American ties. 


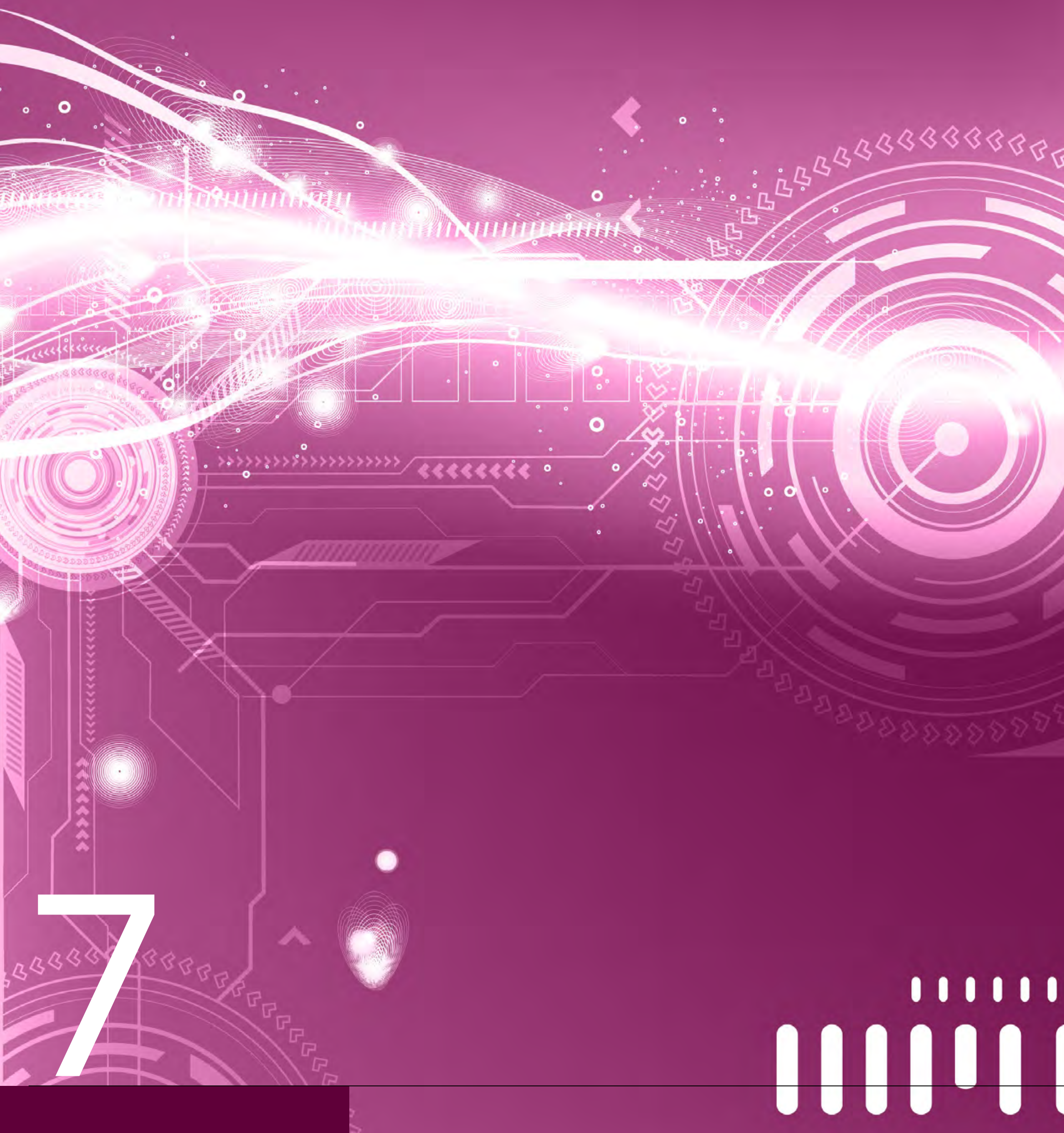

WHAT IS DIGITAL TRANSFORMATION MATURITY? 


\subsection{The What and The How}

The digital transformation framework has many moving parts because much is required to drive a successful digital transformation. However, executives can think about the figure in terms of two main areas (see Figure 15).

The What: The inner boxes, consisting of strategic assets, the nine digital elements, digital capabilities, and investments, are the shape of the transformation. They are the specific set of elements implemented by the organization, and the resources used to do so. Together they represent, in essence, the digital intensity of the organization.

The How: The outer boxes, consisting of digital vision, governance and engagement, are the ways in which leaders will drive the transformation to a successful outcome. They serve as a form of scaffolding through which leaders can ensure that the elements of the "what" are built effectively and that the organization
THE "HOW" IS THE WAY THAT SENIOR EXECUTIVES DRIVE CHANGE THROUGHOUT THE ORGANIZATION. THIS INCLUDES CREATING AND COMMUNICATING VISION, ESTABLISHING GOVERNANCE AND MEASUREMENT MECHANISMS, AND BUILDING A DIGITAL-READY CULTURE.

\section{Transformative Digital Vision}

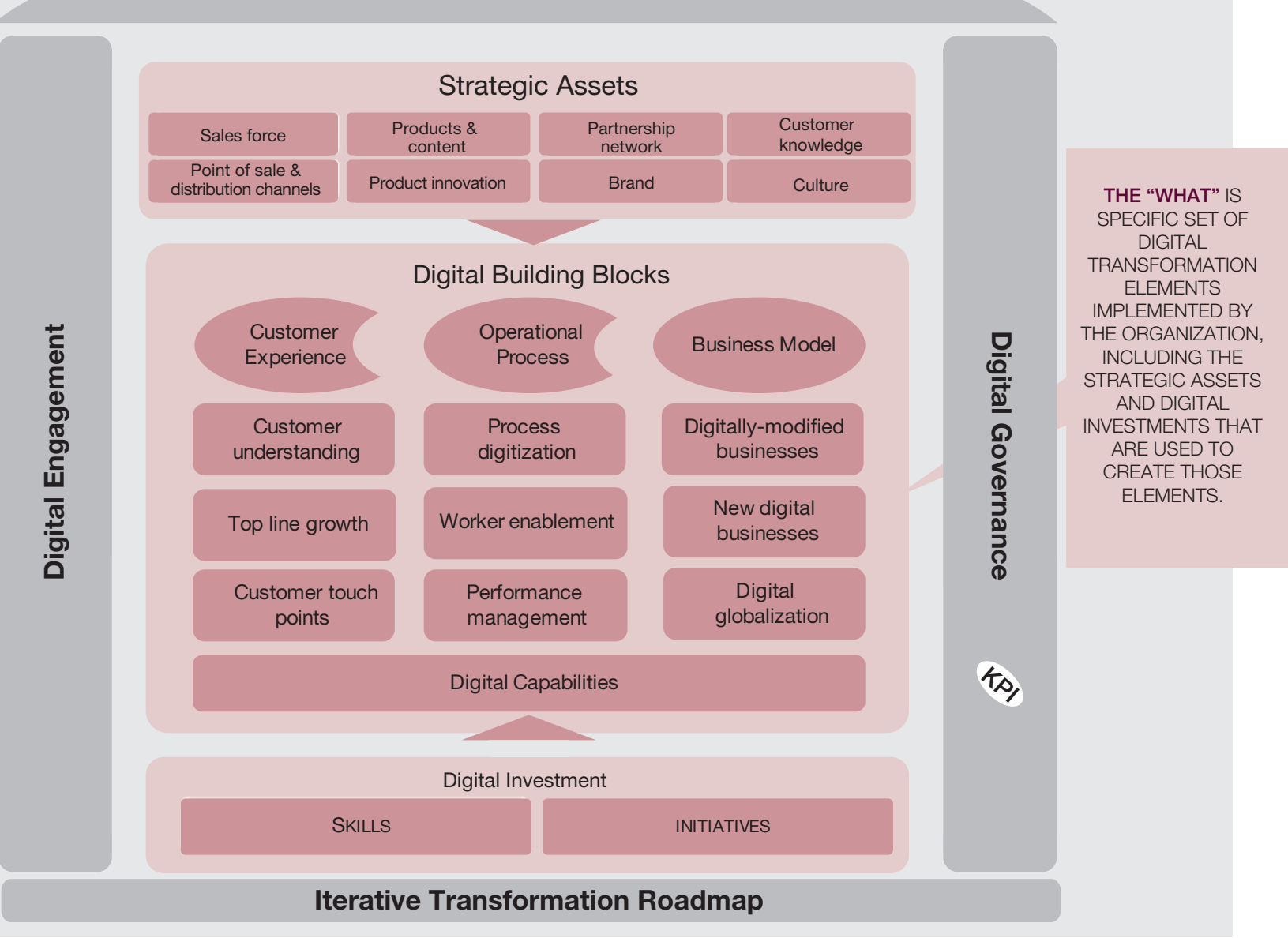


has the skills and culture to drive value from them. It is, in essence, the transformation management intensity of the organization.

Together, the "what" and the "how" represent the digital maturity of an organization (see figure 14). They can be thought about as digital "Style" and "Substance." Firms that are mature on both dimensions can drive powerful digital transformation that yields business value. Unfortunately, many firms in our study are mature at only one, or neither.

\subsection{Digital maturity matrix}

Figure 16 shows digital maturity as a classic $2 \times 2$, highlighting four different types of approaches to driving digital transformation.

Firms in the lower left are Digital Beginners. They are doing very little with advanced digital capabilities, although they have more traditional digital capabilities such as ERP, internet or e-mail. Companies may be in this quadrant by choice. For example, executives in a specialty chemicals firm believe that their B2B customers will not be interested in social media or mobile technologies, and that their engineering employees are very effective with traditional collaboration tools. However, many companies are in this quadrant by accident. They are either unaware of the possibilities of new digital technologies or are starting

\section{FASHIONISTAS}

- Many advanced digital features (such as social, mobile) in silos

- No overarching vision

- Underdeveloped coordination

- Digital culture may exist in silos

\section{BEGINNERS}

- Management skeptical of the business value of advanced digital technologies

- May carry out some experimentation

- Immature digital culture

\section{DIGIRATI}

- Strong overarching digital vision

- Good governance

- Many digital initiatives generating business value in measurable ways

- Strong Digital culture

\section{CONSERVATIVES}

- Overarching digital vision exists, but may be underdeveloped

- Few advanced digital features, though traditional digital capabilities many be mature.

- Strong digital governance across silos

- Taking active steps to build digital skills and culture 
some investments without effective transformation management in place.

Firms at the top left are Digital Fashionistas. They have implemented a large amount of sexy digital "stuff." Some of these items may be creating value, but some may not. While the digital fashion items may look good together, they may clash with other items, and they rarely generate synergies. Digital fashionistas are motivated to bring on digitallypowered change, but their digital transformation strategy is not founded on real knowledge of how to proceed. As a result, fashionistas may look good, but rarely have much substance - in terms of business value - behind the sizzle. We see many fashionistas in B2C businesses, where executives (especially the marketing units) believe they must move fast to keep up with the fast-moving world of consumer electronics, but do not necessarily have a clear vision for how the elements will collectively create value. Some firms are in this quadrant at the enterprise level even though digital efforts in silos may be in other quadrants. This is the case with a hospitality firm, in which marketing and customer service are both relatively mature digitally, but the two are not yet well coordinated.

Firms at the bottom right are Digital

Conservatives. These represent the wise old men and women of the digital world. They understand the need for a strong unifying vision and for governance and internal engagement activities to ensure prudent investment management. However, they are typically skeptical of the value of new trends, sometimes to their detriment. Digital conservatives understand where the company should be going and how to master digital challenges, but they can't always build organizational momentum to carry out an ambitious program. As a result, though aiming to spend wisely, their careful approach may cause them to miss valuable opportunities that their more stylish competitors will pounce on.

Firms at the top right are Digirati. These firms truly understand how to drive value from digital transformation. They combine a strong shared vision for transformation, careful governance and engagement, and sufficient investment in new opportunities. Through managing the "how" carefully, they develop a digital culture that can envision further changes and implement them wisely. Through managing the "what" sufficiently, they continuously advance the competitive advantage they draw from implementing digital transformation.

\subsection{How to assess your digital maturity}

Figure 17 shows how companies in our study scored on an initial qualitative assessment of digital maturity.

What is your digital maturity? Use the guidelines below to assess your company. Note that digital maturity may vary across business units, and will usually be different at the enterprise level than within silos.

- Digital intensity: consider how advanced your digital capabilities are relative to competitors, or relative to rising expectations of customers and employees. How much are you investing in new technologies such as mobile, analytics and social media? Are you launching technology-enabled 
business changes such as locationaware marketing or analyticsinformed supply chain planning? Digital intensity also depends on your firm's IT capabilities and the strategic relationship between IT and non-business managers, since most digital opportunities will have a significant IT component.

- Transformation management intensity: consider how carefully you are envisioning and managing the firm's move into the digital future. Is the vision well-articulated and shared? How well are you governing and coordinating digital investments? How well are you helping the organization be ready for changes?

Using these guidelines, you can plot your own organization on the digital transformation maturity matrix. Then, if your company is not yet a digerati, take steps to address any weaknesses in your digital transformation capabilities or build a roadmap to maneuver up the maturity cycle.

\section{FASHIONISTAS}

\section{DIGIRATI}

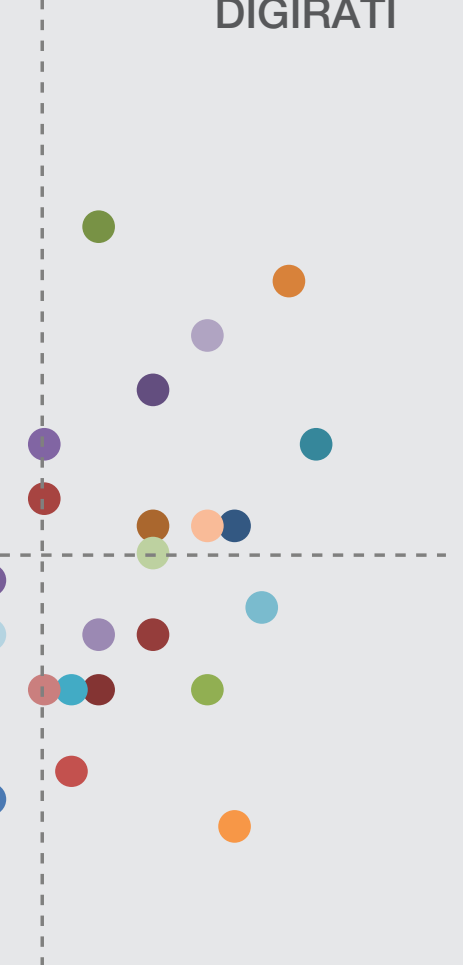

CONSERVATIVES

Note: Digital maturity assessments were conducted by the research team using information from interviews. Vertical and horizontal lines are located at the mean value plus $1 / 2$ standard deviation along each axis.

\section{Transformation management intensity}




\section{Case Study}

\section{PAGES JAUNES: CAPITALIZING ON DIGITAL OPPORTUNITIES}

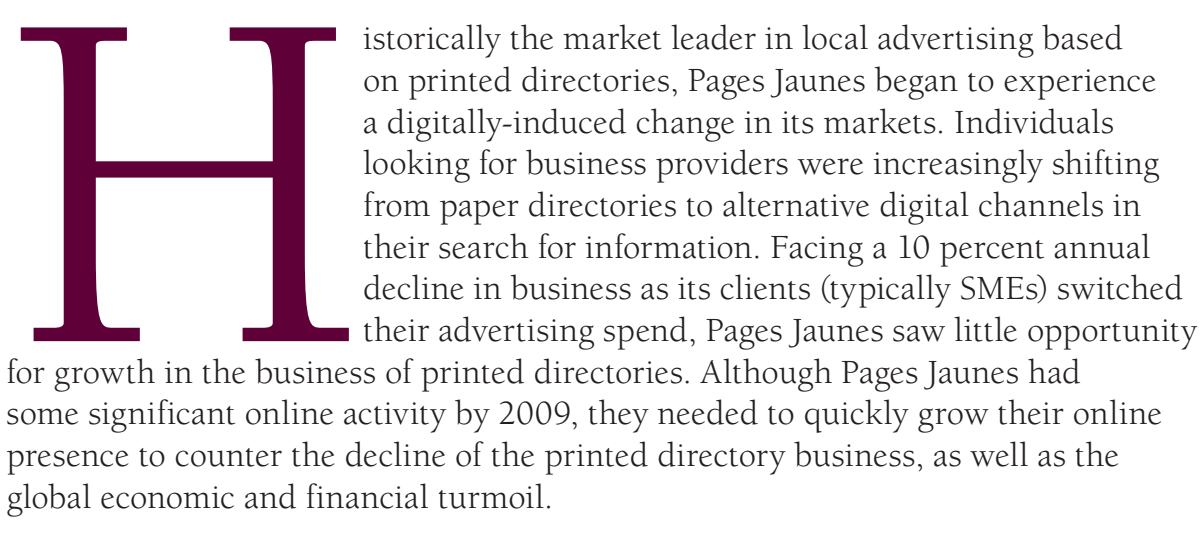

New CEO Jean-Pierre Remy convinced the organization to re-envision its business. Pages Jaunes was not just a provider of directories, but was a trusted name in the relationship business. It disseminated local information to end-users (consumers) and provided local communications services to advertisers. The firm held only a 10 percent share of its newly-envisioned market, providing scope for growth. With this new vision, the company started to identify new client needs and rapidly capture opportunities to extend its relationship brokering role from paper to the Internet.

Pages Jaunes' strategy aimed to accelerate the transformation of the business from having roughly 30 percent of its business online to 75-80 percent in 3-4 years.

Elements of the transformation included:

- restructuring the organization into an SME-facing Advertising group and two consumer-facing -media groups, focused on Internet \& print respectively. A second $\mathrm{CIO}$ was recruited to lead developments around new digital technology while keeping the first $\mathrm{CIO}$ in charge of the existing, and still critical, IT systems.

- recognizing the value of the relationships built over many years between the firm's salesforce and its thousands of clients. The firm committed to keeping as many salespeople as possible and helping them to learn to sell the firm's new services.

- substantial effort to change the culture of an erstwhile state-owned company by recruiting new tech-savvy employees and by instituting KPIs to retain focus on attention and performance.

- educating clients (SMEs) about how digital possibilities could impact their own business and drive revenues, without losing sight of that portion of its clients' customers who continue to rely on the print channel. 
- building content strength online by creating and managing websites for more than 100,000 SMEs in France and Spain. The firm is experimenting with social media possibilities, 3D applications, and focusing on various other services, including display, SEM, SEO, direct marketing, coupons, etc. to reach an increasingly online audience.

- building and continuing to enhance a strong online mobile brand presence, reaching number three in the marketplace after Google and Facebook in France. This tactic allows the firm to spend less than before on driving traffic to its sites (search engine marketing).

- leveraging analytics heavily to analyze audience traffic and patterns, to quantify for each advertising client the value and impact of products they purchase and to help clients optimize media spending.

Pages Jaunes' approach built on an already strong brand, a strong network of salespeople who had existing relationships with SME clients, strong investments in content and therefore the ability to partner (vs. compete) with major search engines like Bing and Google, and with major content sources like Facebook.

Within two years of this strategic shift, Pages Jaunes earned $€ 1$ billion from online sales at a 50 percent net margin. Online business comprised about 50-60 percent of its total business, and the firm was well-positioned to reach its goal of 75-80 percent online business within two years. 


\section{CONCLUSION}

Digital technology, which transformed the media industry, is now transforming the rest of the commercial world. Companies in all industries and regions are experimenting with - and benefiting from - digital transformation. Whether it is in the way individuals work and collaborate, the way business processes are executed within and across organizational boundaries, or in the way the company understands and services customers, digital technology provides a wealth of opportunity to those willing to change their businesses to take advantage of it.

The pressure points for change are increasing from many angles. Globalization is dictating efficient integration of businesses which can only be achieved through digital processes and collaborative tools. Employees and customers are starting to demand new ways of working. As competitors and new entrants make digitally-enabled practices a reality in an industry, other firms will need to follow.

Faced with these challenges, what are the key steps senior executives should take to steer their organization?

\section{Envision the digital future for your firm.}

-What assets will be valuable in a digitally-transformed business?

- How can you transform customer experience? Internal operations? Your business model?

- How can units work differently - and work together differently - in a more connected way?

\section{Invest in digital transformation initiatives}

- Are you getting all of the value out of your previous technology and platform investments in ERP, analytics, or collaboration tools? If not, what is necessary to get the foundations right?
-Where are the key investment areas that will maximize the contribution to the new vision?

- Can you de-risk some of the investments through experimentation and controlled testing?

- What skills are missing in your digital transformation initiatives? Do you need to hire new executives? Retrain front-line employees? Partner with another firm to gain capabilities?

\section{Lead the change from the top}

- How do you communicate the vision and engage the organization on a large scale? How do you monitor engagement?

- What process do you have in place to iterate the vision and strategy?

- How do you coordinate investments and activities across silos? What is the best organizational model to coordinate digital initiatives in parallel to the core business?

- What KPIs and metrics do you need to put in place to monitor the progress of your digital transformation towards your strategic goals?

- What mechanism do you use to make the necessary adjustments?

Digital transformation requires skills and influence that only senior leaders possess. Create a transformative vision that is clear and compelling to galvanize the organization. Mindfully consider what parts of your company should move - and why, and when - to make it implementable. Build digital maturity in two dimensions to increase your chances of success. Then take actions and monitor progress to turn your vision into reality. 


\section{MIT Center for Digital Business and Capgemini Consulting Team members}

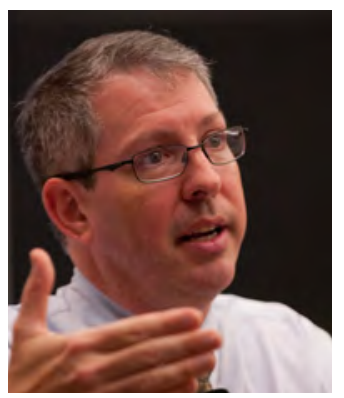

George Westerman, a research scientist in MIT Sloan's Center for Digital Business (CDB), is also faculty chair for the MIT Sloan executive course Transforming Your Business Through Information Technology. George's research examines the role of executive leaders in driving competitive advantage from digital technology. George is co-author of two award-winning books, The Real Business of IT: How CIOs Create and Communicate Value, and IT Risk: Turning Business Threats into Competitive Advantage. Prior to earning his Doctorate from Harvard Business School, he gained more than fifteen years of experience in innovation and technology management. georgew@mit.edu

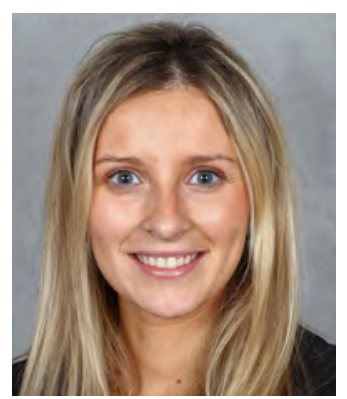

Claire Calméjane is a Managing Consultant in the Technology Transformation Practice. She worked for the last 6 years at the Paris office and is currently visiting scientist at the MIT Center for Digital Business, working with the researchers. She specializes in Digital Transformation and IT Financial Services. She graduated from a Computer Science Engineering School and holds a Master degree from HEC Paris Business School. claire.calmejane@capgemini.com

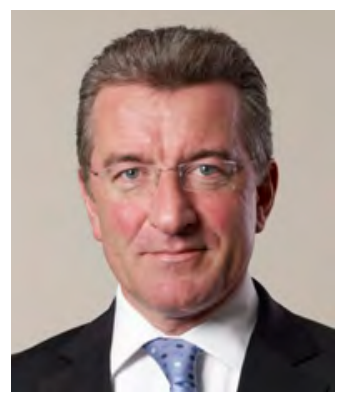

Didier Bonnet is a Managing Director and global head of practices at Capgemini Consulting. Didier has more than 25 years' experience in strategy development, globalisation, internet $\&$ digital economics and business transformation for large multinational corporations and private equity firms.

He has authored several research articles and is regularly quoted in the press e.g., WSJ, FT, the Economist and provides commentary for broadcasters such as the BBC, CNN, Reuters and CNBC.

Didier graduated from a French Business School and holds a DPhil from Oxford University. He is based in London. didier.bonnet@capgemini.com

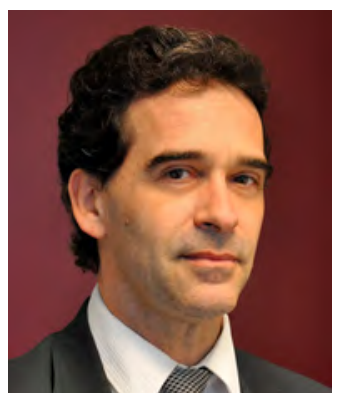

Patrick Ferraris is the Global Leader of the Technology Transformation practice within Capgemini Consulting. With over 20 years of consulting experience, Patrick has supported large multinational organizations in their digital strategy and transformation with a focus in Telecom, Media, Internet, Insurance and Transportation. He is an alumnus from M.I.T and Ecole Nationale des Ponts \& Chaussées.

patrick.ferraris@capgemini.com

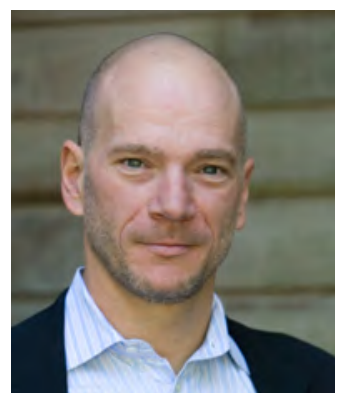

Andrew McAfee, a principal research scientist at MIT, studies the ways that information technology (IT) affects businesses. He coined the phrase "Enterprise 2.0;" his book on the topic was published in 2009 by Harvard Business School Press. He has also held appointments as a professor at Harvard Business School and a fellow at Harvard's Berkman Center for Internet and Society. In 2008, he was named the 38th most influential person in IT.

amcafee@mit.edu 
Special thanks to our other research team members: Greg Gimpel and Mael Tannou.

We would like also to acknowledge the valuable support of a number of colleagues who have either helped with the data collection or have contributed with their ideas and insights; they are: Emmanuel Rilhac, Vincent Leonetti, Julien Pontault, Deborah Soule, Michael Krauch, Cyril Francois, Martin Hanlon, Kieran Draper, Jerome Buvat.

Last thanks to our point of contacts who facilitated access to their clients and often performed interviews: Eric Lamotte, Peter Lindell, Ravouth Keuky, Jesus Viceira Alguacil, John Varghese, Diego Mackee, David Blackwood, Benoit Pradet, Philippe David, Stanislas de Roys, Mark Hoffland, Eric Kruidhof, Ulf Holmgren, Philippe Bigot, Jari Matula, Adeline Pairault, Sebastien Blot, Sandra Lagrue and others. 


\section{About Capgemini Consulting}

Capgemini Consulting is the Global Strategy and Transformation Consulting brand of the Capgemini group, specializing in advising and supporting organizations in transforming their business, from the development of innovative strategy through to execution, with a consistent focus on sustainable results. Capgemini Consulting proposes to leading companies and governments a fresh approach which uses innovative methods, technology and the talent of over 4,000 consultants worldwide

For more information: www.capgemini-consulting.com

Follow us on Facebook: http://www.facebook.com/capgemini.consulting.global

Follow us on Twitter: @CapgeminiConsul

\section{Capgemini Consulting}

\section{About the MIT Center for Digital Business}

Founded in 1999, the MIT Center for Digital Business (http://digital.mit.edu) joins leading companies, visionary educators, and some of the best students in the world together in inventing and understanding the business value made possible by digital technologies. We are supported entirely by corporate sponsors with whom we work in a dynamic interchange of ideas, analysis, and reflection intended to solve real problems. The Center has funded more than 50 faculty and performed more than 75 research projects focused on understanding the impact of technology on business value, and developing tools and frameworks our sponsors can use for competitive advantage.

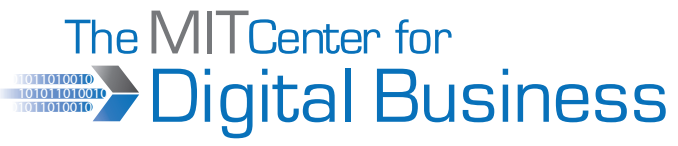

